\title{
Wavelet Galerkin pricing of American options on Lévy driven assets
}

\section{Report}

\section{Author(s):}

Matache, Ana-Maria; Nitsche, Pal-Andrej; Schwab, Christoph

Publication date:

2003-07

Permanent link:

https://doi.org/10.3929/ethz-a-004570535

Rights / license:

In Copyright - Non-Commercial Use Permitted

Originally published in:

SAM Research Report 2003-06 


\title{
Wavelet Galerkin Pricing of American Options on Lévy Driven Assets *
}

\author{
A.-M. Matache, P.-A. Nitsche and C. Schwab**
}

Research Report No. 2003-06

July 2003

Seminar für Angewandte Mathematik

Eidgenössische Technische Hochschule

CH-8092 7ïrich

Switzerland

${ }^{*}$ Research was supported by Credit Suisse Group, Swiss Re and UBS AG through RiskLab, Switzerland under the RiskLab project Fast Deterministic Valuations for Assets Driven by Lévy Processes

${ }^{* *}$ Work performed while C. Schwab was staying at the Isaac Newton Institute of Mathematical Sciences under the programme "Computational Challenges in Partial Differential Equations" 


\title{
Wavelet Galerkin Pricing of American Options on Lévy Driven Assets *
}

\author{
A.-M. Matache, P.-A. Nitsche and C. Schwab** \\ Seminar für Angewandte Mathematik \\ Eidgenössische Technische Hochschule \\ CH-8092 Zürich \\ Switzerland
}

Research Report No. 2003-06

July 2003

\begin{abstract}
,
The price of an American style contract on assets driven by Lévy processes with infinite jump activity is expressed as solution of a parabolic variational integro-differential inequality (PIDI). A Galerkin discretization in logarithmic price using a wavelet basis is presented with compression of the moment matrix of the jump part of the price process' Dynkin operator. An iterative solver with wavelet preconditioning for the resulting large matrix inequality problems is presented and its efficiency is demonstrated by numerical experiments.
\end{abstract}

Keywords: Lévy processes, integro-differential operators, variational inequalities, Galerkin discretization, bi-orthogonal wavelet basis, wavelet preconditioning

${ }^{*}$ Research was supported by Credit Suisse Group, Swiss Re and UBS AG through RiskLab, Switzerland under the RiskLab project Fast Deterministic Valuations for Assets Driven by Lévy Processes

${ }^{* *}$ Work performed while C. Schwab was staying at the Isaac Newton Institute of Mathematical Sciences under the programme "Computational Challenges in Partial Differential Equations" 


\section{Introduction}

In asset pricing, models beyond the classical Black-Scholes (B-S) have been proposed for the stochastic dynamics of the risky asset: we mention only stochastic volatility models and 'stochastic clocks'. The latter lead to so-called jump-diffusion price processes: the Wiener process in the B-S model is replaced by a Lévy process (see e.g. [31, 3, 17, 30, 11, 8, 9] and $[5,35]$ and the references there for background information on Lévy processes). Such processes allow more realistic modelling of the price dynamics for the risky asset (see e.g. [17]). The inclusion of jumps into the asset price dynamics has been investigated for several years - let us mention here only [2] and the references there. In almost all papers we are aware of, the jumps in the price process have been modelled as finite intensity processes, as e.g. a Poisson process, i.e. in any finite time interval only a finite number of large jumps occur. In the early 90ies, however, processes with infinite intensity have been proposed. We mention here only the Variance Gamma (VG) [31, 30] and the so-called CGMY models [11].

For pricing European Vanilla contracts on assets with Lévy price processes, the explicitly available characteristic function allows to apply Fourier-Laplace transformations for the solution of the pricing problem. This approach has been used e.g. in [12].

For American style contracts with such price processes, the transformation methods do not seem to be generally applicable. We mention that the analytical tool of Wiener-Hopf factorization allows, at least for infinite horizon problems, to derive semi-analytical solutions also for American style contracts [10]. The exercise boundary and the solution of the free boundary value problem can be expressed in terms of the factors from the Wiener-Hopf factorization. These factors are, except for some particular cases, not known explicitly, and in general very difficult to compute. Moreover, these methods fail for time-dependent or local volatility models, or for finite maturity. Also, general pay-off functions arising in American style digital contracts are not easily handled by such semi-analytical approaches.

The analysis and implementation of fast, deterministic pricing schemes for American style contracts on assets driven by general Lévy processes is the purpose of the present paper. Our approach is based on finite element solution of the associated deterministic differential equation. This equation involves the Dynkin operator of the semigroup generated by the price process which for Lévy processes is an integro-differential operator. Upon discretization, the linear systems to be solved in each implicit time step have dense and ill-conditioned stiffness matrices. We propose here a spline wavelet basis for discretization in the (logarithmic) price variable which allows to 'compress' these matrices to sparse, well-conditioned ones that can be inverted by iterative schemes in almost linear complexity while not affecting the accuracy of the computed prices. For American style contracts, the wavelet basis allows to precondition the iterative solver for the associated Linear Complementarity Problems (LCPs) in each time step. The algorithm allows to treat any Lévy price process, even pure jump processes with infinite jump intensity. Moreover, general pay-off functions are admissible allowing in particular to handle compound options, with contracts of European or American style. For infinite activity jump processes, the process' Lévy measure has a density with respect to the Lebesgue measure which is nonintegrable and must be interpreted in the sense of distributions. We present a variational framework for the associated parabolic integro-differential inequality which accomodates nonintegrable Lévy densities. The integro-differential inequality is discretized using the backward Euler scheme in time and a wavelet-based finite element discretization in (logarithmic) price. We show that using the wavelet type basis functions, the condition number of the resulting large matrix inequality problems remains bounded independent of the discretization level. This allows to conclude the convergence of a fixed point type iteration with a rate 
which is independent of the meshwidth.

The outline of the paper is as follows: in Section 2, we present the admissible price processes. Section 3 contains the formulation of the American style pricing problem and the derivation of the price as solution of a parabolic integro-differential inequality. Section 4 discusses the discretization of the inequality - here, we show that the moment matrix due to the nonlocal part of the parabolic integrodifferential operator can be compressed to an approximate, sparse matrix while still having bounded condition number. Some a-priori error bounds for the numerical solution are also stated. In Section 5, we then address the numerical solution of the large complementarity problems in each time-step. We prove that a fixed point iteration converges at a rate which is independent of the discretization parameter and give a generalization of Cryer's algorithm in the wavelet basis to locate the exercise boundary. Section 6 contains numerical results obtained with our approach. They indicate in particular the failure of the smooth fit principle in certain pure jump price models.

\section{Price Processes}

\subsection{Lévy processes}

Let $\left(\Omega, \mathcal{F},\left(\mathcal{F}_{t}\right)_{0<t<\infty}, \mathbf{P}\right)$ be a filtered probability space satisfying the usual hypothesis. Let $X=\left(X_{t}\right)_{0 \leq t<\infty}$ with $X_{0}=0$ a.s. be a Lévy process, i.e., a process with stationary, independent increments that is stochastically continuous $[5,35]$.

The Lévy-Khintchine formula describes explicitly a Lévy process in terms of its Fourier transform $E_{\mathbb{Q}}\left[e^{-i u X_{t}}\right]$ under a chosen equivalent martingale measure $\mathbb{Q}$ :

$$
E_{\mathbb{Q}}\left[e^{-i u X_{t}}\right]=e^{-t \psi(u)}
$$

for some function $\psi$ called the Lévy exponent of $X$. It has the following representation

$$
\begin{aligned}
\psi(u)=\frac{\sigma^{2}}{2} & u^{2}+i \alpha u \\
& +\int_{|x|<1}\left(1-e^{-i u x}-i u x\right) \nu_{\mathbb{Q}}(\mathrm{d} x)+\int_{|x|>1}\left(1-e^{-i u x}\right) \nu_{\mathbb{Q}}(\mathrm{d} x)
\end{aligned}
$$

for some $\sigma, \alpha \in \mathbb{R}$ and for a measure $\nu_{\mathbb{Q}}$ on $\mathbb{R} \backslash\{0\}$ satisfying

$$
\int_{\mathbb{R}} \min \left(1, x^{2}\right) \nu_{\mathbb{Q}}(\mathrm{d} x)<\infty .
$$

The Lévy measure $\nu_{\mathbb{Q}}(\mathrm{d} x)$ measures the arrival rate of jumps of size $x$. The Lévy-triple $\left(\sigma, \alpha, \nu_{\mathbb{Q}}\right)$ completely determines $X_{t}$ and the characteristic exponent $\psi$ is related to the symbol of the nonlocal operator $L_{X}^{\mathbb{Q}}$ which is the infinitesimal generator of the transition semi-group of $X_{t}$ under the chosen equivalent martingale measure $\mathbb{Q}[35,5]$. We assume here that the equivalent martingale measure $\mathbb{Q}$ has been chosen by some procedure, we refer to $\lceil 15,16,19,13\rceil$ and the references therein for varions results in this direction.

\subsection{Variance Gamma Process}

The variance gamma process $[30,31\rceil$ is a Brownian motion with drift in which the calendaristic time has been changed to a 'business' time modeled by a gamma process $\gamma(t ; \nu)$ with mean rate unity and variance rate $\nu$

$$
X_{V G}(t ; \sigma, \nu, \theta)=\theta \gamma(t ; \nu)+\sigma W_{\gamma(t ; \nu)} .
$$


From the density of the gamma process

$$
f_{\gamma(t ; \nu)}(x)=\frac{x^{t / \nu-1} e^{-x / \nu}}{\nu^{t / \nu} \Gamma(t / \nu)}
$$

one obtains the characteristic function of the gamma process

$$
\phi_{\gamma(t ; \nu)}(u)=E\left[e^{i u \gamma(t ; \nu)}\right]=\left(\frac{1}{1-i \nu u}\right)^{t / \nu}
$$

and the characteristic function of the variance gamma process has the form

$$
\phi_{X_{V G}(t ; \sigma, \nu, \theta)}(u)=E\left[e^{i u X_{V G}(t ; \sigma, \nu, \theta)}\right]=\left(\frac{1}{1-i \theta \nu u+\sigma^{2} \nu u^{2} / 2}\right)^{t / \nu} .
$$

This expression of the characteristic function $\phi_{X_{V G}(t ; \sigma, \nu, \theta)}$ together with

$$
\frac{1}{1-i \theta \nu u+\sigma^{2} \nu u^{2} / 2}=\frac{1}{1-i \eta_{p} u} \cdot \frac{1}{1+\eta_{n} u}
$$

where

$$
\eta_{p}=\sqrt{\frac{\theta^{2} \nu^{2}}{4}+\frac{\sigma^{2} \nu}{2}}+\frac{\theta \nu}{2}, \quad \eta_{n}=\sqrt{\frac{\theta^{2} \nu^{2}}{4}+\frac{\sigma^{2} \nu}{2}}-\frac{\theta \nu}{2}
$$

lead to another interpretation of the variance gamma process, namely as the difference of two independent gamma processes

$$
X_{V G}(t ; \sigma, \nu, \theta) \stackrel{\text { law }}{=} \gamma_{p}\left(t ; \eta_{p} / \nu, \eta_{p}^{2} / \nu\right)-\gamma_{n}\left(t ; \eta_{n} / \nu, \eta_{n}^{2} / \nu\right) .
$$

The representation (2.7) of the variance gamma process and the representation of the Lévy density for the gamma process lead to the following form of the Lévy density for the VG process

$$
k_{V G}(x)= \begin{cases}\frac{1}{\nu} \cdot \frac{e^{-\frac{1}{\eta_{n}}|x|}}{|x|} & \text { if } \quad x<0 \\ \frac{1}{\nu} \cdot \frac{e^{-\frac{1}{\eta_{p}}|x|}}{|x|} & \text { if } \quad x>0\end{cases}
$$

The VG process is a process of infinite activity, i.e., $\int_{\mathbb{R}} k_{V G}(x) \mathrm{d} x=+\infty$.

\subsection{CGMY process}

The CGMY process [11] generalizes the VG process by adding a new parameter in the Lévy density that allows the resulting Lévy process to have both finite or infinite activity and finite or infinite variation. Specifically, the Lévy density of the CGMY process is given by

$$
k_{C G M Y}(x)= \begin{cases}C \frac{e^{-G|x|}}{|x|^{1+Y}} & \text { if } \quad x<0 \\ C \frac{e^{-M|x|}}{|x|^{1+Y}} & \text { if } \quad x>0,\end{cases}
$$

where $C>0, G, M>0$ and $Y<2$. The case $Y=0$ is the special case of the variance gamma process. 
The parameter $C$ is related to the overall level of activity, $G$ and $M$ control the exponential rate of decay at $\mp \infty$ of the Lévy density and lead to skewed distributions if they are unequal. If $G=M$ one obtains Lévy processes from the Koponen family [26]. Empirical evidence however indicates that the probability density functions of returns are almost symmetric at the origin but that the left tails are fatter than the right ones. For $G<M$ indeed the left tail of the distribution of $X_{t}$ is heavier than the right tail consistently with the distribution implied from the option prices. The parameter $Y$ is related to the fine structure of the stochastic process. Remarkably, the characteristic function of the CGMY process is available in closed form [11].

\section{American Option Pricing}

Our purpose is the valuation of American-style options, i.e., options that can be exercised at any time up to the expiration date $T$, on an underlying with price process $X_{t}$. Most listed stock options, including those on European exchanges, are American-style options. The early exercise feature makes their valuation more complex than that of European-style options in a Black-Scholes world. It also implies higher prices for an American-style option than for a European contract with the same price process. We formulate the pricing problem of an American-style contract as optimal stopping problem for $X_{t}$ and express prices as solutions of parabolic variational integro-differential inequalities. To accomodate general pay-off functions which may grow polynomially at infinity, these inequalities are set in Sobolev spaces with exponential weights. This setting is the basis for numerical solution methods in the next sections.

\subsection{Optimal stopping problem}

Consider the price $f\left(S_{t}, t\right)$ of an American option with expiry date (maturity) $T$ when the risk-neutral dynamics of the risky asset $S_{t}$ are given by the following geometric law:

$$
S_{t}=S_{0} e^{\left(r+c-\sigma^{2} / 2\right) t+X_{t}}
$$

where $X_{t}$ is a Lévy process of the form $X_{t}=\sigma W_{t}+Y_{t}$, with $W_{t}$ denoting the Brownian motion and $Y_{t}$ being a quadratic pure jump Lévy process independent of $W_{t}$ as in Section 2. The correction parameter $c$ in (3.1) ensures that the mean rate of return on the asset is risk-neutrally $r$, i.e. that $e^{-c t}=E_{\mathbb{Q}}\left[e^{Y_{t}}\right]$. Let $g(S)$ denote a pay-off function of the option (conditions on $g$ shall be discussed below). The problem of optimal exercising is equivalent to an optimal stopping problem and the value $f\left(S_{t}, t\right)$ of the contract is given by

$$
f\left(S_{t}, t\right)=\sup _{t \leq \tau \leq T} E_{\mathbb{Q}}\left[e^{-r(\tau-t)} g\left(S_{\tau}\right) \mid \mathcal{F}_{t}\right],
$$

where the supremum is taken over all stopping times $\tau$ on the probability space generated by the asset price process. Equation (3.2) means that the owner chooses the optimal exercise policy to maximize the expected discouted pay-off.

Remark 3.1 For the American put $g(S)=(K-S)_{+}$, with $K>0$ being the strike price, and for each $t$ there exists a critical value $S_{t}^{*}$ such that for all $S_{t} \leq S_{t}^{*}$ the value of the American put option is the value of immediate exercise, i.e., $f\left(t, S_{t}\right)=g\left(S_{t}\right)$, while for $S_{t}>S_{t}^{*}$ the value exceeds the immediate exercise value. The curve $S_{t}^{*}$ is referred to as the critical exercise boundary, the region $\mathcal{C}=\left\{(t, S) \mid S>S_{t}^{*}\right\}$ is called the continuation region and the complement $\mathcal{E}$ of $\mathcal{C}$ is the exercise region. For the detailed study of the free boundary problem for the American put in the Brownian case, see e.g. [36], Karatzas and Shreve (1998) [25], Musiela and Rutkowski (1997) [33]. 


\subsection{Parabolic integro-differential inequality}

Let $\mu(\mathrm{d} x, \mathrm{~d} t)$ denote the integer valued random measure (the jump measure) that counts the number of jumps of $Y_{t}$ in space-time. By stationarity of Lévy processes, the compensator of the measure $\mu(\mathrm{d} x, \mathrm{~d} t)$ has the form $\nu_{\mathbb{Q}}(\mathrm{d} x) \times \mathrm{d} t$, with $\mathrm{d} t$ being the Lebesgue measure. In the following we will assume that the Lévy measure $\nu_{\mathbb{Q}}(\mathrm{d} x)$ has a density $k_{\mathbb{Q}}$, the Lévy kernel, with respect to Lebesgue measure so that $\nu_{\mathbb{Q}}(\mathrm{d} x)=k_{\mathbb{Q}}(x) \mathrm{d} x$ and we will drop the subscript $\mathbb{Q}$.

Remark 3.2 By (3.1), (2.1)-(2.2) and by $E_{\mathbb{Q}}\left[S_{t}\right]<\infty$ we obtain that $E_{\mathbb{Q}}\left[e^{X_{t}}\right]=e^{-t \psi(i)}<\infty$, with $\psi$ being the Lévy exponent in (2.2). As a consequence, the Lévy density $k$ has to satisfy both the integrability condition (2.3) and $\int_{|x| \geq 1} e^{x} k(x) \mathrm{d} x<\infty$. For the case of the CGMYmodel (2.9) these integrability conditions for the Lévy density imply that $Y<2$ and $M>1$.

For sufficiently regular $g$ and for $\sigma \neq 0$ the price $f$ in (3.2) is known to satisfy the following parabolic integro-differential inequality (see, e.g. [4])

$$
\begin{aligned}
& \frac{\partial f}{\partial t}(t, S)+\frac{\sigma^{2}}{2} S^{2} \frac{\partial^{2} f}{\partial S^{2}}(t, S)+r S \frac{\partial f}{\partial S}(t, S)-r f(t, S) \\
& \quad+\int_{\mathbb{R}}\left[f\left(t, S e^{x}\right)-f(t, S)-S \frac{\partial f}{\partial S}(t, S)\left(e^{x}-1\right)\right] \nu(\mathrm{d} x) \leq 0 \\
& f(t, S) \geq g(S) \\
& (f(t, S)-g(S))\left(\frac{\partial f}{\partial t}(t, S)+\frac{\sigma^{2}}{2} S^{2} \frac{\partial^{2} f}{\partial S^{2}}(t, S)+r S \frac{\partial f}{\partial S}(t, S)-r f(t, S)\right. \\
& \left.\quad+\int_{\mathbb{R}}\left[f\left(t, S e^{x}\right)-f(t, S)-S \frac{\partial f}{\partial S}(t, S)\left(e^{x}-1\right)\right] \nu(\mathrm{d} x)\right)=0 \\
& f(T, S)=g(S) .
\end{aligned}
$$

We also mention [10] for theoretical results on the relation between the solution of the free boundary value problem and that of the optimal stopping for the case of the perpetual American put, i.e., when $T=\infty$.

For numerical treatment, we change to logarithmic price $x=\ln (S) \in \mathbb{R}$ and time to maturity $\tau-T-t$ and introduce $u(\tau, x)-f\left(T-\tau, e^{x}\right)$. If we denote by $\psi(x)-g\left(e^{x}\right)$, the resulting parabolic integro-differential inequality for the value function $u$ reads

$$
\begin{aligned}
& \frac{\partial u}{\partial \tau}+\mathcal{A}_{\mathrm{B}-\mathrm{S}}[u]+\mathcal{A}_{\text {jump }}[u] \geq 0 \quad \text { in }(0, T) \times \mathbb{R} \\
& u(\tau, x) \geq \psi(x) \quad \text { a.e. in }[0, T] \times \mathbb{R} \\
& (u(\tau, x)-\psi(x))\left(\frac{\partial u}{\partial \tau}+\mathcal{A}_{\mathrm{B}-\mathrm{S}} u+\mathcal{A}_{\text {jump }}[u]\right)=0 \quad \text { in }(0, T) \times \mathbb{R} \\
& u(0, x)=\psi(x),
\end{aligned}
$$

where the infinitesimal generator (or Dynkin operator) of the transition semi-group of $X_{t}$ is given by $\mathcal{A}=\mathcal{A}_{\mathrm{B}-\mathrm{S}}+\mathcal{A}_{\text {jump }}$ with

$$
\begin{aligned}
& \mathcal{A}_{\mathrm{B}-\mathrm{S}}[\varphi]=-\frac{\sigma^{2}}{2} \frac{\mathrm{d}^{2} \varphi}{\mathrm{d} x^{2}}+\left(\frac{\sigma^{2}}{2}-r\right) \frac{\mathrm{d} \varphi}{\mathrm{d} x}+r \varphi \\
& \mathcal{A}_{\text {jump }}[\varphi]=-\int_{\mathbb{R}}\left(\varphi(x+y)-\varphi(x)-\left(e^{y}-1\right) \frac{\mathrm{d} \varphi}{\mathrm{d} x}(x)\right) k(y) \mathrm{d} y .
\end{aligned}
$$

Remark 3.3 Unless explicitly stated otherwise, we assume in the following that the price process has a non-zero diffusion component, i.e. $\sigma \neq 0$. 


\subsection{Variational formulation}

Of particular interest will be American Put contracts where the pay-off is $g(S)=(K-S)_{+}$. The pay-off in $\log$-price variable $x=\log (S)$ is given by

$$
\psi(x)=\left(K-e^{x}\right)_{+} .
$$

We note in passing that all our results apply to more general pay-off functions $\psi(x)$ with polynomial growth as $|x| \rightarrow \infty$ as well.

Our pricing algorithm will be based on a Galerkin discretization of (3.4)-(3.7) in the logarithmic price $x=\log (S)$. This Galerkin discretization will be based on a variational formulation of (3.4)-(3.7). Since the pay-off $\psi$ does not decay as $|x| \rightarrow \infty$, we use Sobolev spaces with exponential weights, see also [23] for this technique in the Brownian case: for $\mu, \nu>0$ define

$$
\eta(x):= \begin{cases}\nu x & \text { if } x<0 \\ \mu x & \text { if } x>0\end{cases}
$$

The weighted Sobolev spaces with exponent $\eta$ are given by

$$
H_{\eta}^{j}(\mathbb{R}):=\left\{v \in L_{1 \circ \mathrm{c}}^{1}(\mathbb{R}) \mid \frac{\mathrm{d}^{k} v}{\mathrm{~d} x^{k}} e^{\eta} \in L^{2}(\mathbb{R}) \forall k=0,1, \ldots, j\right\} .
$$

We introduce the bilinear form $a^{\eta}(\cdot, \cdot)$ corresponding to the space operator $\mathcal{A}$ : for $\varphi, \phi \in C_{0}^{\infty}(\mathbb{R})$ we define

$$
\begin{aligned}
a^{\eta}(\varphi, \phi) & =a_{\mathrm{B}-\mathrm{S}}^{\eta}(\varphi, \phi)+a_{\text {jump }}^{\eta}(\varphi, \phi):=\int_{\mathbb{R}} \mathcal{A}[\varphi](x) \phi(x) e^{2 \eta(x)} \mathrm{d} x \\
& =-\frac{\sigma^{2}}{2} \int_{\mathbb{R}}\left(\frac{\mathrm{d}^{2} \varphi}{\mathrm{d} x^{2}}(x)-\frac{\mathrm{d} \varphi}{\mathrm{d} x}(x)\right) \phi(x) e^{2 \eta(x)} \mathrm{d} x-r \int_{\mathbb{R}}\left(\frac{\mathrm{d} \varphi}{\mathrm{d} x}(x)-\varphi(x)\right) \phi(x) e^{2 \eta(x)} \mathrm{d} x \\
& -\int_{\mathbb{R}} \int_{\mathbb{R}}\left\{\varphi(x+y)-\varphi(x)-\frac{\mathrm{d} \varphi}{\mathrm{d} x}(x)\left(e^{y}-1\right)\right\} k(y) \phi(x) e^{2 \eta(x)} \mathrm{d} y \mathrm{~d} x .
\end{aligned}
$$

The following theorem implies the well-posedness of the integro-differential inequality. It shows that $a^{\eta}(\cdot, \cdot)$ can be extended continuously to $H_{\eta}^{1}(\mathbb{R}) \times H_{\eta}^{1}(\mathbb{R})$.

Theorem 3.4 Assume that the Lévy density $k(y)$ has exponential tails and the exponent $\eta$ in (3.9) satisfies $\mu<\nu$ and $\int_{\mathbb{R}} e^{-\eta(y)}|y| k(y) \chi_{\{|y| \geq 1\}} \mathrm{d} y<+\infty$. Then $a^{\eta}(\cdot, \cdot)$ can be extended continuously to a bounded bilinear form on $H_{\eta}^{1}(\mathbb{R}) \times H_{\eta}^{1}(\mathbb{R})$. Moreover, $a^{\eta}(\cdot, \cdot)$ is coercive on $H_{\eta}^{1}(\mathbb{R}) \times H_{\eta}^{1}(\mathbb{R})$. More precisely, there exist $\alpha_{\eta}, \beta_{\eta}>0$ and $C_{\eta}>0$ such that

$$
\begin{aligned}
\left|a^{\eta}(\varphi, \phi)\right| & \leq C_{\eta}\|\varphi\|_{H_{\eta}^{1}(\mathbb{R})}\|\phi\|_{H_{\eta}^{1}(\mathbb{R})} \quad \forall \varphi, \phi \in H_{\eta}^{1}(\mathbb{R}) \\
a^{\eta}(\varphi, \varphi) & \geq \alpha_{\eta}\|\varphi\|_{H_{\eta}^{1}(\mathbb{R})}^{2}-\beta_{\eta}\|\varphi\|_{L_{\eta}^{2}(\mathbb{R})}^{2} \quad \forall \varphi \in H_{\eta}^{1}(\mathbb{R}) .
\end{aligned}
$$

The proof of this assertion is given in Appendix A.

In what follows we identify the bilinear form defined in $(3.10)$ with its extension to $H_{\eta}^{1}(\mathbb{R}) \times$ $H_{\eta}^{1}(\mathbb{R})$. Note that $\psi \in H_{\eta}^{1}(\mathbb{R})$ for all $\mu, \nu>0$.

Admissible solutions for the variational formulation of (3.4) $-(3.7)$ will be sought in the convex cone

$$
\mathcal{K}_{\psi}:=\left\{v \in H_{\eta}^{1}(\mathbb{R}) \mid v \geq \psi \text { a.e. } x\right\} .
$$


The variational formulation of the parabolic integro-differential inequality (3.4)-(3.7) reads: Find $u \in L^{2}\left((0, T) ; H_{\eta}^{1}(\mathbb{R})\right), \frac{\partial u}{\partial \tau} \in L^{2}\left((0, T) ; L_{\eta}^{2}(\mathbb{R})\right)$ such that $u(\tau, \cdot) \in \mathcal{K}_{\psi}$ almost everywhere in $(0, T)$ and such that for all $v \in H_{\eta}^{1}(\mathbb{R}) \cap \mathcal{K}_{\psi}$

$$
\begin{aligned}
& \left(\frac{\partial u}{\partial \tau}, v-u\right)_{L_{\eta}^{2}(\mathbb{R})}+a^{\eta}(u, v-u) \geq 0 \quad \text { a.e. in }\left(0, I^{\prime}\right), \\
& u(0, \cdot)=\psi .
\end{aligned}
$$

If $\mathcal{A} \psi \in L_{\eta}^{2}$, by (3.11) and (3.12) the variational inequality (3.13)-(3.14) admits a unique solution $u$ (see [20], Chapter 6, Section 2) and it holds

$$
u, \frac{\partial u}{\partial t} \in L^{2}\left(0, T ; H_{\eta}^{1}\right) \cap L^{\infty}\left(0, T ; L_{\eta}^{2}\right)
$$

Since $u \in H_{\eta}^{1}(\mathbb{R})$ with $\eta$ as in $(3.9), u$ decays exponentially at $+\infty$. Heuristically, at $-\infty$ the exact boundary condition is $\psi(-\infty)=K$.

For the numerical solution, we therefore localize this problem on a bounded domain $\Omega_{R}=$ $(-R, R), R>0$ sufficiently large, with homogeneous essential boundary conditions at $\pm R$.

\subsection{Localization}

To prepare the numerical solution of (3.13)-(3.14), we solve for the the excess to pay-off function

$$
U=u-\psi \in\left\{v \in H_{\eta}^{1}(\mathbb{R}) \mid v \geq 0 \text { a.e. } x\right\}
$$

rather than for $u$. We also restrict the range of admissible prices to a bounded domain $\Omega_{R}=$ $(-R, R)$, with $R>0$ sufficiently large and solve for $U^{R}(\tau, \cdot) \in \mathcal{K}_{0}:=\left\{v \in H_{0}^{1}\left(\Omega_{R}\right) \mid v \geq 0\right\}$, $U^{R} \in L^{2}\left((0, T) ; H_{0}^{1}\left(\Omega_{R}\right)\right), \frac{\partial U}{\partial \tau} \in L^{2}\left((0, T) ; L^{2}\left(\Omega_{R}\right)\right)$ such that

$$
\begin{aligned}
& \left(\frac{\mathrm{d} U^{R}}{d \tau}, v-U^{R}\right)_{L^{2}\left(\Omega_{R}\right)}+a\left(U^{R}, v-U^{R}\right) \geq-a\left(\psi, v-U^{R}\right) \\
& U^{R}(0, \cdot)=0 \quad \text { a.e. in }(0, T), \quad \forall v \in V \cap \mathcal{K}_{0}
\end{aligned}
$$

where $V:=H_{0}^{1}\left(\Omega_{R}\right)$ and the bilinear form $a(\varphi, \psi)$ is, for all $\varphi, \psi \in H_{0}^{1}\left(\Omega_{R}\right)$, given by

$$
\begin{aligned}
a(\varphi, \phi) & =-\frac{\sigma^{2}}{2} \int_{\Omega_{R}}\left(\frac{\mathrm{d}^{2} \varphi}{d x^{2}}(x)-\frac{\mathrm{d} \varphi}{\mathrm{d} x}(x)\right) \phi(x) \mathrm{d} x \\
& -r \int_{\Omega_{R}}\left(\frac{\mathrm{d} \varphi}{\mathrm{d} x}(x)-\varphi(x)\right) \phi(x) \mathrm{d} x \\
& -\int_{\Omega_{R}} \int_{\mathbb{R}}\left\{\varphi(x+y)-\varphi(x)-\frac{\mathrm{d} \varphi}{\mathrm{d} x}(x)\left(e^{y}-1\right)\right\} k(y) \phi(x) \mathrm{d} y \mathrm{~d} x .
\end{aligned}
$$

To simplify the notation, we drop the superscript $R$ from $U^{R}$, i.e., we denote by $U=U^{R}$.

Proposition 3.5 Theorem 3.4 holds with $a^{\eta}$ replaced by a and $H_{\eta}^{1}(\mathbb{R})$ replaced by $H_{0}^{1}\left(\Omega_{R}\right)$. With obvious notations, $a=a_{\mathrm{B}-\mathrm{S}}+a_{\mathrm{jump}}$ and there exists a positive constant $C=C(R)>0$ such that for all $\varphi \in H_{0}^{1}\left(\Omega_{R}\right)$

$$
a_{\mathrm{B}-\mathrm{S}}(\varphi, \varphi) \geq C \sigma^{2}\|\varphi\|_{H^{1}\left(\Omega_{R}\right)}^{2}, \quad a_{\mathrm{jump}}(\varphi, \varphi) \geq 0 .
$$

For a proof, see e.g. Remark 3.6 in [32]. Due to these properties, (3.16)-(3.17) admits a unique solution. For the pure jump case $\sigma=0$, the parabolic problem still has eigenvalues with the correct real part, so that our solution algorithm below remains stable also in this limiting case. 


\section{Discretization}

Since closed form solutions of (3.13), (3.14) are not available in general, numerical solutions of the pricing problem are necessary. To this end, we discretize (3.16)-(3.17) by a Finite Element (FE) method in $\Omega_{R}$ and by the backward Euler scheme in time, reducing it to a sequence of matrix inequality problems. Since $\mathcal{A}_{\text {jump }}$ is nonlocal and unbounded, its stiffness matrix is densely populated and ill-conditioned.

Using a wavelet basis of the corresponding finite dimensional space, we show that the matrix for $\mathcal{A}_{\text {jump }}$ can be 'compressed', i.e. approximated by a sparse and well conditioned matrix without compromising accuracy.

\subsection{Time stepping}

We discretize (3.16)-(3.17) by a Finite Element (FE) method in $\Omega_{R}$ and by the backward Euler scheme in time, reducing it to a sequence of matrix inequality problems.

Let $k=T / M$, with $M \in \mathbb{N}$ be a time step. Let us denote by $U^{m}, m=0,1, \ldots, M$ the solution to the following backward Euler discretization of $(3.16)-(3.17)$ :

Find $U^{m+1} \in V \cap \mathcal{K}_{0}, m=0,1, \ldots, M-1$, such that

$$
\begin{aligned}
& \left(\underline{\partial} U^{m}, v-U^{m+1}\right)_{L^{2}\left(\Omega_{R}\right)}+a\left(U^{m+1}, v-U^{m+1}\right) \geq-a\left(\psi, v-U^{m+1}\right) \\
& \text { a.e. in }(0, T), \quad \forall v \in V \cap \mathcal{K}_{0} \\
& U^{0}=0 .
\end{aligned}
$$

Here we denoted by $\underline{\partial}$ the finite difference operator $\underline{\partial} U^{m}:=\left(U^{m+1}-U^{m}\right) / k$.

\subsection{Space discretization. LCP}

The sequence (3.16)-(3.17) of elliptic variational inequalities can be reduced to a sequence of Linear Complementarity Problems (LCPs) by restricting $V$ to a finite dimensional subspace $V_{N}$.

As in Finite Element approaches to the B-S equation, we use spaces $V_{N}$ of continuous piecewise linear functions with respect to a equidistant subdivision $\mathcal{T}:-R=x_{0}<x_{1}<\cdots<x_{N+1}=R$ of the truncated domain $\Omega_{R}$ :

$$
V_{N}=\operatorname{span}\left\{v(x) \in V:\left.v\right|_{\left(x_{i-1}, x_{i}\right)}, x_{i} \in \mathcal{T} \text {, is linear }\right\} .
$$

The Finite Element (FE) formulation to (4.1)-(4.2) reads:

Find $U_{N}^{m}:(0, T) \rightarrow V_{N} \cap \mathcal{K}_{0}$ such that.

$$
\begin{aligned}
& \left(\underline{\partial} U_{N}^{m}, v-U_{N}^{m+1}\right)_{L^{2}\left(\Omega_{R}\right)}+a\left(U_{N}^{m+1}, v-U_{N}^{m+1}\right) \geq-a\left(\psi, v-U_{N}^{m+1}\right) \\
& \text { a.e. in }(0, T), \quad \forall v \in V_{N} \cap \mathcal{K}_{0} \\
& U_{N}^{0}=0 .
\end{aligned}
$$

The approximate solutions $U_{N}^{m}$ converge, as $N \rightarrow \infty$, to the exact solution: by a result of Johnson [24] there holds, under some mild restriction on the active set where $U_{N}^{m}$ vanishes which usually holds in the pricing of American put contracts, the error estimate

$$
\max _{m}\left\|u^{m}-U_{N}^{m}\right\|_{L^{2}(\Omega)}+\left(\sum_{m=1}^{M} k\left\|u^{m}-U_{N}^{m}\right\|_{H^{1}(\Omega)}^{2}\right)^{1 / 2} \leq C\left(k^{3 / 4}|\log k|^{1 / 4}+h\right)
$$


which indicates convergence as the time-steps are reduced $(k \rightarrow 0)$ or as the meshwidth $h=$ $2 R / N$ in the price variable tends to zero.

The sequence of finite dimensional variational inequalities (4.3)-(4.4) corresponds to a sequence of matrix variational inequalities which we now derive.

Let $\mathcal{B}=\left\{\Phi_{j}\right\}_{j=1}^{N}$ be a basis of $V_{N}$, i.e. $V_{N}=\operatorname{Span}\left(\Phi_{j}\right)_{j=1}^{N}$ and denote by $\mathbf{M}$ the mass matrix with respect to $\mathcal{B}$ and by $\mathbf{A}$ the stiffness matrix of $a(\cdot, \cdot)$ with respect to $\mathcal{B}$, i.e., $M_{i, j}=$ $\left(\Phi_{i}, \Phi_{j}\right)_{L^{2}\left(\Omega_{R}\right)}, A_{i, j}=a\left(\Phi_{j}, \Phi_{i}\right)$. Notice that the stiffness matrix $\mathbf{A}$ is, due to the nonlocal Dynkin operator, nonsparse.

We denote likewise by $\underline{F}$ the load vector with components $F_{j}=-a\left(\psi, \Phi_{j}\right)$ and by $\underline{v}$ the coefficient vector of the $\mathrm{FE}$ function $v \in V_{N}$ with respect to $\mathcal{B}$.

Then coefficient vectors $\underline{v}$ of $\mathrm{FE}$ functions $v$ in $V_{N} \cap \mathcal{K}_{0}$ are vectors in $\mathbb{R}^{N}$ satisfying componentwise

$$
\mathbf{C} \underline{v} \geq 0
$$

where $\mathbf{C}$ stands for the change of basis matrix from $\mathcal{B}$ into the canonical 'hat' function basis $\left\{\Psi_{j}\right\}_{j=1}^{N}$ with $\Psi_{j}(x)=\max \left(0,1-\left|x-x_{j}\right| / h\right)$.

With these notations, (4.3)-(4.4) can be equivalently rewritten as a sequence of LCPs:

Find $\underline{U}_{N}^{m} \in \underline{\mathcal{K}}_{0}:=\left\{\underline{v} \in \mathbb{R}^{N} \mid \mathbf{C} \underline{v} \geq 0\right\}, m=0,1, \ldots, M$, such that

$$
\left(\underline{v}-\underline{U}_{N}^{m+1}\right)^{\top}(\mathbf{M}+k \mathbf{A}) \underline{U}_{N}^{m+1} \geq\left(\underline{v}-\underline{U}_{N}^{m+1}\right)^{\top}\left(k \underline{F}+\mathbf{M} \underline{U}_{N}^{m}\right) \quad \forall \in \underline{v} \in \underline{\mathcal{K}}_{0} .
$$

\subsection{Wavelet basis}

Rather than the classical "hat" functions $\Psi_{j}(x)=\max \left(0,1-\left|x-x_{j}\right| / h\right)$, we choose as basis $\mathcal{B}$ of $V_{N}$ biorthogonal spline wavelets with a larger support. These slightly more involved shape functions serve two purposes: first, in the wavelet basis the bilinear form $a(\cdot, \cdot)$ will correspond to a matrix where most elements are negligible, yielding an approximate bilinear form $\tilde{a}(\cdot, \cdot)$ and a "compressed", sparse matrix $\tilde{\mathbf{A}}$ with only $N \log N$ nonvanishing entries. The error introduced by this matrix compression is not larger than the error due to Galerkin discretization [34].

Second, the wavelet basis will also allow optimal preconditioning. Perturbed bilinear forms $\widetilde{a}$ are obtained by various matrix compression techniques which reduce the dense matrices $\mathbf{A}$ to sparse ones which can be manipulated in linear complexity [34].

To define the wavelets, we consider dyadic partitions $\mathcal{T}_{L}$ of $\Omega_{R}$ into $N+1=2^{L}$ subintervals of equal size. We set $N=N_{L}$ and denote $V_{N}$ by $V_{L}$ to indicate the dependence on the subdivision level $L$.

We use piecewise linear, continuous biorthogonal wavelets $\psi_{j}^{l}$ that in the interior of $\Omega_{R}$ have values $0, \ldots 0,-1,2,-1,0, \ldots, 0$. In the case of Dirichlet conditions the values are $0,2,-1,0, \ldots, 0$ (and similarly at the right boundary), see Figure 1 . Note that the boundary wavelets do not have vanishing moments.

The support of wavelet $\psi_{j}^{l}$ is denoted by $S_{j}^{l}:=\operatorname{supp} \psi_{j}^{l}$. It has diameter bounded by $C 2^{-l}$. Wavelets $\psi_{j}^{l}$ with $\bar{S}_{j}^{l} \cap \partial \Omega_{K}=\emptyset$ have vanishing moments up to order 1 , i.e.,

$$
\left(\psi_{j}^{l}, 1\right)=\left(\psi_{j}^{l}, x\right)=0
$$

The functions $\psi_{j}^{l}$ for $l \geq l_{0}$ are obtained by scaling and translation of the generating wavelets $\psi_{j}^{1}, j=0,1,2$ shown in Figure 1. Any $v \in V_{L}$ has the representation

$$
v(x)=\sum_{l=0}^{L} \sum_{j=1}^{M^{l}} v_{j}^{l} \psi_{j}^{l}(x)
$$



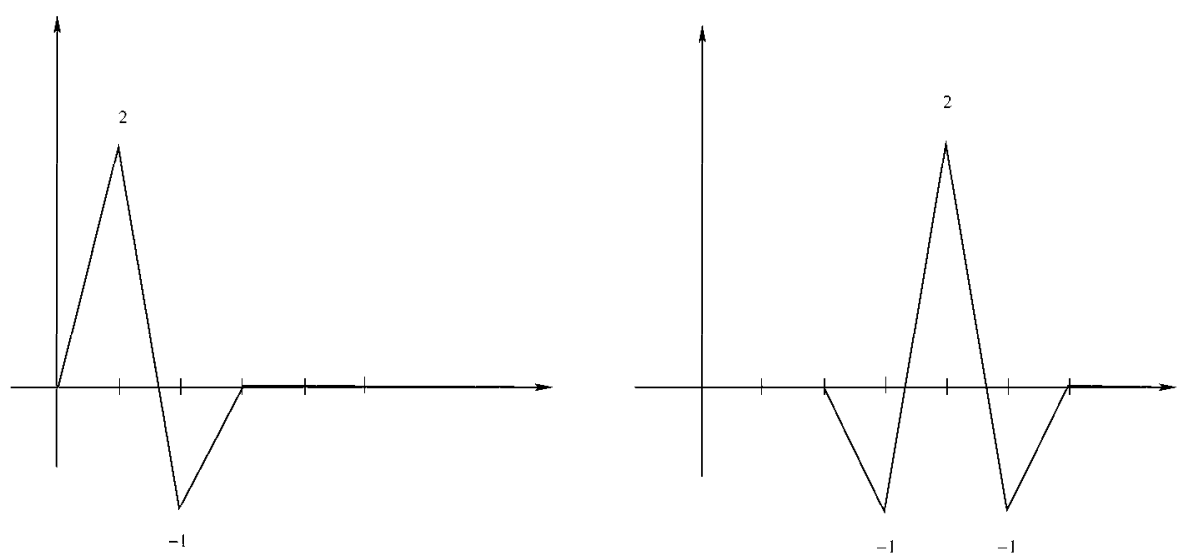

Figure 1: Generating wavelets: interior wavelets (right) and boundary wavelets for Dirichlet boundary conditions (left).

with $v_{j}^{l}=\left(v, \tilde{\psi}_{j}^{l}\right)$ where $\tilde{\psi}_{j}^{l}$ are the so-called dual wavelets (note that in a Galerkin scheme these dual basis functions never enter explicitily).

Any $v \in V$ can be written as infinite series

$$
v(x)=\sum_{l=0}^{\infty} \sum_{j=1}^{M^{l}} v_{j}^{l} \psi_{j}^{l}(x)
$$

with $v_{j}^{l}=\left(v, \tilde{\psi}_{j}^{l}\right)$ which converges in $\tilde{H}^{\theta}\left(\Omega_{R}\right):=\left\{\left.v\right|_{\Omega_{R}}\left|v \in H^{\theta}(\mathbb{R}), v\right|_{\mathbb{R} \backslash \Omega_{R}}=0\right\}$ for $0 \leq \theta \leq 1$. For $v \in V$ we can define a projection $\Gamma_{L}: V \rightarrow V_{L}$ by truncating the wavelet expansion in the log-price variable

$$
P_{L} v:=\sum_{l=0}^{L} \sum_{j=1}^{M^{l}} v_{j}^{l} \psi_{j}^{l}(x)
$$

For preconditioning our LCP solver, we exploit the norm equivalence

$$
\forall v \in \tilde{H}^{\theta}\left(\Omega_{R}\right): \quad c_{1}\|v\|_{\tilde{H}_{\theta}}^{2} \leq \sum_{l=0}^{\infty} \sum_{j=1}^{M^{l}}\left|v_{l}^{j}\right|^{2} 2^{2 l \theta} \leq c_{2}\|v\|_{\tilde{H}_{\theta}}^{2}, \quad 0 \leq \hat{\theta} \leq 1 .
$$

\subsection{Matrix compression}

The bilinear form $a$ on $V_{L} \times V_{L}$ in the wavelet basis corresponds to a matrix $\mathbf{A}$ with elements $A_{(l, j),\left(l^{\prime}, j^{\prime}\right)}=a\left(\psi_{j}^{l}, \psi_{j^{\prime}}^{l^{\prime}}\right)$. The Lévy density of $\mathcal{A}_{\text {jump }}$ satisfies the Calderón-Zygmund type estimate

$$
\left|D_{y}^{m} k(y)\right| \leq C(m)|y|^{-(1+Y+m)} \quad \forall m \in \mathbb{N}_{0}, \forall y \neq 0
$$


(everything that follows holds also for more general densities $k(x, y)$ which satisfy $(4.10)$ uniformly with respect to $x$; such densities arise for homogeneous 'diffusions with jumps' $X_{t}$ in the sense of Definition 2.18 in [22]). Relation (4.10) implies the decay of the matrix elements with increasing distance of the supports of corresponding wavelets. To be specific, we define the compressed matrix $\tilde{\mathbf{A}}$ and the corresponding bilinear form $\tilde{a}$ by setting certain small matrix elements in $\mathbf{A}$ to zero: with $A_{(j, l),\left(j^{\prime}, l^{\prime}\right)}=a\left(\psi_{j}^{l}, \psi_{j^{\prime}}^{l^{\prime}}\right)$, we set

$$
\tilde{A}_{(j, l),\left(j^{\prime}, l^{\prime}\right)}:= \begin{cases}A_{(j, l),\left(j^{\prime}, l^{\prime}\right)} & \text { if } \operatorname{dist}\left(S_{j}^{l}, S_{j^{\prime}}^{l^{\prime}}\right) \leq \delta_{l, l^{\prime}} \text { or } S_{j}^{l} \cap \partial \Omega_{R} \neq \emptyset \\ 0 & \text { otherwise }\end{cases}
$$

where $S_{j}^{l}:=\operatorname{supp}\left(\psi_{j}^{l}\right)$. Here the truncation parameters $\delta_{l, l^{\prime}}$ are given by

$$
\delta_{l, l^{\prime}}:=\kappa \max \left\{2^{-L+\hat{\alpha}\left(2 L-l-l^{\prime}\right)}, 2^{-l}, 2^{-l^{\prime}}\right\}
$$

The meaning of $\kappa>0$ and $\hat{\alpha}>0$ in (4.11)-(4.12) is as follows: the wavelet basis $\psi_{j}^{l}$ implies a block structure of the matrix $\mathbf{A}$. The compressed matrix $\tilde{\mathbf{A}}$ obtained from (4.11) retains only diagonals of each block $\mathbf{A}_{l, l^{\prime}}$ resulting in the typical 'finger-band' structure of waveletcompressed stiffness matrices (see [32] and the references there). In (4.12), the parameter $\kappa$ governs the bandwidth in the largest block $\tilde{\mathbf{A}}_{L, L}$ of $\tilde{\mathbf{A}}$ which is fixed independently of $L$ while $\hat{\alpha}$ governs the growth of this bandwidth in the blocks $\tilde{\mathbf{A}}_{l, l^{\prime}}$ with $l+l^{\prime}<2 L$, see Figure 2 .
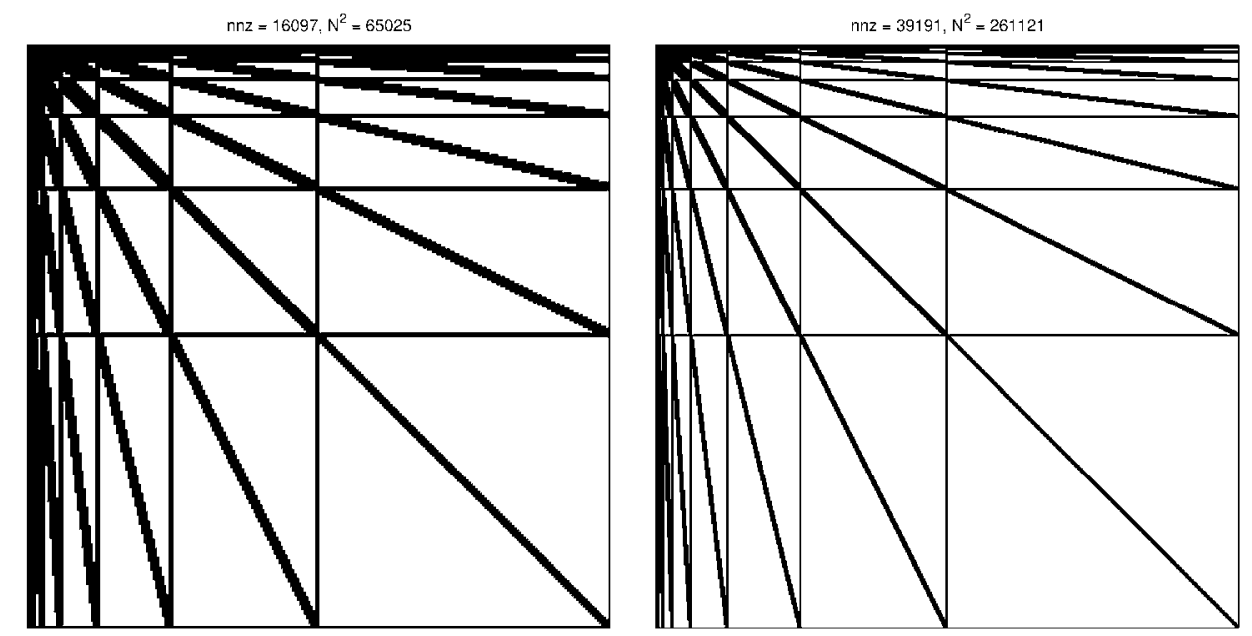

Figure 2: Sparsity pattern of the compressed matrix $\tilde{A}$ in wavelet basis; compression parameters: $\kappa=1.0, \hat{\alpha}=0.8$; CGMY parameters: $C=1.0, Y=1.4, G=0.4, M=1.6 ; L=7$ (left) and $L=8$ (right).

If the truncation parameters $\hat{\alpha}$ and $\kappa$ are suitably chosen, the corresponding perturbation in the bilinear forms is small. To quantify this, we need to consider functions in $V$ which have additional regularity and introduce for this purpose the spaces $\mathcal{H}^{s}\left(\Omega_{R}\right)$ which are defined as

$$
\mathcal{H}^{s}\left(\Omega_{R}\right)=\left\{\begin{array}{c}
V \quad \text { for } s=1 \\
V \cap H^{s}\left(\Omega_{R}\right) \quad \text { for } s>1 .
\end{array}\right.
$$

Proposition 4.1 [34] For any $\delta>0$ there exists $\kappa$ in (4.12) sufficiently large such that for all $L>0$ holds

$$
|a(u, v)-\widetilde{a}(u, v)| \leq \delta\|u\|_{H^{1}\left(\Omega_{R}\right)}\|v\|_{H^{1}\left(\Omega_{R}\right)} \quad \forall u, v \in V_{L}
$$


If additionally $u \in \mathcal{H}^{2}\left(\Omega_{R}\right)$, then for

$$
\hat{\alpha} \geq \frac{4}{4+Y}
$$

holds

$$
\left|a\left(P_{L} u, v\right)-\widetilde{a}\left(P_{L} u, v\right)\right| \leq C h|\log h|^{\nu}\|u\|_{\mathcal{H}^{2}\left(\Omega_{R}\right)}\|v\|_{H_{0}^{1}\left(\Omega_{R}\right)} \quad \forall v \in V_{L}
$$

holds with $\nu=\frac{3}{2}$ if equality holds in (4.14), and $\nu=\frac{1}{2}$ otherwise.

If $\hat{\alpha}<1$ in (4.12), then the number of nonzero entries in $\tilde{\mathbf{A}}$ is bounded by $C N_{L} \log N_{L}$. In the case $Y=0$ (which corresponds to the VG process), $\hat{\alpha}=1$ and the above result still holds with at most $C N_{L}\left(\log N_{L}\right)^{2}$ nonzero entries.

We see that for $1 \geq \hat{\alpha} \geq 4 /(4+Y)$ in (4.14), the matrix $\mathbf{A}_{\text {jump }}$ can be compressed to a matrix $\tilde{\mathbf{A}}$ with $O(N \log N)$ nonzero entries with small difference between the bilinear forms.

Remark 4.2 If $\sigma>0$ the price process contains a diffusion component and the order of the operator $\mathcal{A}$ is 2 . In the pure jump case, $\sigma=0$ and the order of $\mathcal{A}$ is, in general, $\max \{1, Y\}$. The consistency result of Proposition 4.1 can be adapted to this case (with respect to a different scale of spaces), see [34] for a proof.

Lel $0 \leq \omega \leq 1$ and define $\tilde{H}^{\omega}\left(\Omega_{R}\right):=\left\{\left.u\right|_{\Omega_{R}}: u \in H^{\omega}(\mathbb{R})\right.$ and $\left.\left.u\right|_{\mathbb{R} \backslash \Omega_{R}}=0\right\}$. For $\omega \neq 1 / 2 \mathrm{il}$ holds $\tilde{H}^{\omega}\left(\Omega_{R}\right)=H_{0}^{\omega}\left(\Omega_{R}\right)$ which is defined as the closure of $C_{0}^{\infty}\left(\Omega_{R}\right)$ with respect to the norm in $H^{\omega}\left(\Omega_{R}\right)$.

Proposition 4.3 Assume that $X_{t}$ is a pure jump Lévy process (i.e. $\sigma=0$ ) of CGMY type with density $k$ as in (2.9) with $0<Y<2$. Then there exist positive constants $\alpha=\alpha(R)>0$ and $\beta=\beta(R)>0$ such that

$$
a(u, u) \geq \alpha\|u\|_{\tilde{H}^{Y / 2}\left(\Omega_{R}\right)}^{2}-\beta\|u\|_{L^{2}\left(\Omega_{R}\right)}^{2},
$$

i.e., the bilinear form $a(\cdot, \cdot)$ satisfies a Gärding inequality in $\tilde{H}^{Y / 2}\left(\Omega_{R}\right)$.

Proof. It holds that [32]

$$
a(u, u)=\left(\mathcal{A}_{\mathrm{jump}}[u], u\right)=\frac{1}{2} \int_{\Omega_{K}} \int_{\Omega_{K}}(u(x)-u(y))^{2} k(y-x) \mathrm{d} y \mathrm{~d} x .
$$

Writing $k=k_{0}+\left(k-k_{0}\right)$ with $k_{0}(y)=C /|y|^{1+Y}$ we obtain that

$$
a(u, u)=\frac{C}{2} \int_{\Omega_{R}} \int_{\Omega_{R}} \frac{(u(x)-u(y))^{2}}{|x-y|^{1+Y}} \mathrm{~d} x \mathrm{~d} y+\frac{1}{2} \int_{\Omega_{R}} \int_{\Omega_{R}}(u(x)-u(y))^{2}\left(k-k_{0}\right)(y-x) \mathrm{d} y \mathrm{~d} x .
$$

Then the first term in (4.18) above is the $\tilde{H}^{Y / 2}\left(\Omega_{R}\right)$ semi-norm of $u$ [1] and the second double integral corresponds to a compaci, lower order periurbation.

Remark 4.4 In the pure jump case, i.e., when $\sigma=0$ and for $X_{t}$ being a Lévy process of CGMY type it holds

$$
\begin{aligned}
\mathcal{A}[u](x) & =\mathcal{A}_{\text {jump }}[u](x)=-\int_{\mathbb{R}} u^{\prime \prime}(x+y) k^{(-2)}(y) \mathrm{d} y \\
& +c_{1}(Y ; G, M) u^{\prime}(x)+c_{0}(Y ; G, M) u(x), \quad \forall u \in C_{0}^{\infty}\left(\Omega_{R}\right)
\end{aligned}
$$


where

$$
k^{(-2)}(x)=\text { p.f. } \int_{0}^{x} k(y)(x-y) \mathrm{d} y
$$

with integral to be understood in the finite part sense and

$$
c_{1}(Y ; G, M)=\int_{0}^{\infty} \frac{e^{x}-1}{x^{1+Y}}\left(e^{-M x}-e^{-(G+1) x}\right) \mathrm{d} x, \quad c_{0}(Y ; G, M)=\text { p.f. } \int_{0}^{\infty} \frac{e^{-M x}+e^{-G x}}{x^{1+Y}} \mathrm{~d} x .
$$

For $Y \in(1,2), \tilde{H}^{Y / 2}\left(\Omega_{R}\right)=H_{0}^{Y / 2}\left(\Omega_{R}\right)$ and $\left(u^{\prime}, v\right)$ can be understood as duality between $H^{Y / 2-1}\left(\Omega_{R}\right) \cong\left(H^{1-Y / ?}\left(\Omega_{R}\right)\right)^{*}$ and $H^{1-Y / 9}\left(\Omega_{R}\right)$, since $H^{1-Y / ?}\left(\Omega_{R}\right) \hookleftarrow H_{0}^{Y / 2}\left(\Omega_{R}\right)$. As a consequence it holds

$$
|a(u, v)| \leq C\|u\|_{H^{Y / 2}\left(\Omega_{R}\right)}\|v\|_{H^{Y / 2}\left(\Omega_{R}\right)},
$$

i.e., the bilinear form $a(\cdot, \cdot)$ is continuous on $V \times V$ with $V=H_{0}^{Y / 2}\left(\Omega_{R}\right)$.

If $Y \in[0,1)$, the operator $\partial_{\tau}+\mathcal{A}_{\text {jump }}$ is, in general, of hyperbolic type. If $Y=1$, its formal type is neither parabolic nor hyperbolic. If $\sigma=0$ and $Y<1$ discontinuous wavelet approximations are admissible and the advection term $\partial_{x} u$ can be stably discretized by an upwinding Finite Volume Method (FVM).

Remark 4.5 For $Y<0$, i.e. for finite intensity jump processes, compression to $O(N \log N)$ can not be accomplished with the wavelets shown in Figure 1. In this case, discontinuous wavelets with more vanishing moments than (4.7) allow once more compression to $O(N \log N)$ entries while preserving full accuracy of the scheme.

Remark 4.6 We considered only piecewise linear wavelets of degree $p=1$. Results analogous to Proposition 4.1 are also available for wavelets of degree $p>1$ and we refer to [34] for details. We finally remark that a more refined criterion than (4.11) allows compression to $O(N)$ nonzero entries in certain cases while keeping (4.15).

Using $\tilde{a}$ in place of $a$ in (4.21) gives the following sequence of perturbed matrix variational ineqalities

$$
\left(\underline{v}-\underline{\tilde{U}}_{N}^{m+1}\right)^{\top}(\mathbf{M}+k \tilde{\mathbf{A}}) \underline{\tilde{U}}_{N}^{m+1} \geq\left(\underline{v}-\underline{\tilde{U}}_{N}^{m+1}\right)^{\top}\left(k \underline{F}+\mathbf{M} \underline{\tilde{U}}_{N}^{m}\right) \quad \forall \in \underline{v} \in \underline{\mathcal{K}}_{0} .
$$

Proposition 4.1 indicates that as the mesh is refined the prices obtained from (4.21) stay close to those obtained by solving (4.6).

\section{Solution Algorithm}

The matrix variational inequality (4.21) requires the solution of a linear complementarity problem (LCP) in each time step. Standard methods like PSOR (projected SOR) [14] and PSSOR (projected symmetrized SOR) are not suitable, since these methods are preferred when the matrix is symmetric or diagonally dominant. Unlike as in the BS case, in the Lévy case symmetry of the matrix can not be achieved by transformations, since the Lévy densities have in general asymmetric tails.

Our solution algorithm is described first in a general framework, since it is also applicable to other models as e.g. BS models with stochastic volatility. It relies on a fixed point (outer) iteration where in each step a projection onto the convex cone $\underline{\mathcal{K}}_{0}$ has to be realized (inner iteration). Owing to the norm equivalence (4.9) of the wavelet basis, the outer fixed point iteration applied to (4.21) converges at a rate independent of the dimension $N$ of the FE discretization. In each outer iteration, one must realize the $H^{1}\left(\Omega_{R}\right)$ (or an equivalent) projection $P_{\mathcal{K}_{0}}$ onto $\underline{\mathcal{K}}_{0}$. We realize this projection based on a wavelet generalization of the classical Cryer algorithm [14]. 


\subsection{Outer iteration}

\subsubsection{Fixed point iteration}

We describe an iterative solution algorithm for an abstract elliptic variational inequality set in a Hilbert space $\left(\mathcal{V},(\cdot, \cdot)_{\mathcal{V}}\right)$. Let $\|\cdot\|_{\mathcal{V}}$ denote the corresponding norm $\left(\|v\|_{\mathcal{V}}=(v, v)_{\mathcal{V}}^{1 / 2}\right)$ and let $\mathcal{K} \subset \mathcal{V}$ be a closed, convex cone in $\mathcal{V}$. Without loss of generality it can be assumed that $0 \in \mathcal{K}$. Let $u \in \mathcal{K}$ be the solution of the following variational inequality

$$
\text { Find } u \in \mathcal{K} \quad: \quad b(u, v-u) \geq l(v-u) \quad \forall v \in \mathcal{K} .
$$

The bilinear form $b: \mathcal{V} \times \mathcal{V} \rightarrow \mathbb{R}$ is assumed continous and coercive and the linear form $l: \mathcal{V} \rightarrow \mathbb{R}$ is continuous with respect to $\|\cdot\|_{\mathcal{V}}$, i.e. there exist constants $C>0$ and $\alpha>0$ such that for all $v, w \in \mathcal{V}$

$$
b(v, v) \geq \alpha\|v\|_{\mathcal{V}}^{2}, \quad|b(v, w)| \leq C\|v\|_{\mathcal{V}}\|w\|_{\mathcal{V}}, \quad|l(v)| \leq C\|v\|_{\mathcal{V}} .
$$

Let $\langle\cdot, \cdot\rangle_{\mathcal{V}}$ be an inner product on $\mathcal{V}$ equivalent to $(\cdot, \cdot)_{\mathcal{V}}$. Since for each $v \in \mathcal{V}$ it holds that $b(v, \cdot) \in \mathcal{V}^{*}$, Riesz' theorem applies and there exists $B: \mathcal{V} \rightarrow \mathcal{V}^{*}$ and $b_{l} \in \mathcal{V}$ such that

$$
b(v, w)=\langle B v, w\rangle_{\mathcal{V}}, \quad l(v)=\left\langle b_{l}, v\right\rangle_{\mathcal{V}} \quad \forall v, w \in \mathcal{V} .
$$

The variational inequality (5.1) translates into

$$
\text { Find } u \in \mathcal{K} \quad: \quad\langle B u, v-u\rangle_{\mathcal{V}} \geq\left\langle b_{l}, v-u\right\rangle_{\mathcal{V}} \quad \forall v \in \mathcal{K} \text {. }
$$

Denote by \|\|$\cdot\|\|_{\mathcal{V}}$ the norm corresponding to $\langle\cdot, \cdot\rangle_{\mathcal{V}}$. By our assumptions on $b(\cdot, \cdot)$, there are $C_{1}>0, C_{2}>0$ such that for all $v, w \in \mathcal{V}$ it holds

$$
\left|\langle B v, w\rangle_{\mathcal{V}}\right| \leq C_{1}\left|\left\|v\left|\left\|\left.\right|_{\mathcal{V}}|||w|\right\|_{\mathcal{V}}, \quad\langle B v, v\rangle_{\mathcal{V}} \geq C_{2}\right|\right\| v\right| \|_{\mathcal{V}}^{2} .
$$

Let us denote by $P_{\mathcal{K}}$ the $\langle\cdot, \cdot\rangle_{\mathcal{V}}$ projection onto the convex set $\mathcal{K}$. Solving (5.2) is equivalent to solving the fix-point problem [29]

$$
u=\mathcal{S} u:=P_{\mathcal{K}}\left(u-\rho\left(B u-b_{l}\right)\right), \quad \rho>0
$$

We solve (5.4) iteratively:

$$
\text { Given } u_{0} \in \mathcal{V} \text { define } u_{n+1}:=P_{\mathcal{K}}\left(u_{n}-\rho\left(B u_{n}-b_{l}\right)\right) \quad \forall n \geq 0 .
$$

Then $u_{n} \rightarrow u$ as $n \rightarrow \infty$ provided that $0<\rho<2 C_{2} /\left(C_{1}\right)^{2}$, since in this range of $\rho$ the operator $\mathcal{S}$ is non-expanding. The optimal choice is $\rho_{\text {opt }}=C_{2} /\left(C_{1}\right)^{2}$, for which $\left\|\mid \mathcal{S} u_{1}-\mathcal{S} u_{2}\right\|_{\mathcal{V}} \leq$ $q\left\|u_{1}-u_{2}\right\| \mathcal{v}$, with $q:=1-\left(C_{2}\right)^{2} /\left(C_{1}\right)^{2}<1$. Note that the rate of convergence of the fix-point iteration depends only on the constants $C_{1}, C_{2}$ in (5.3)

\subsubsection{Discretization}

We apply the fix-point iteration $(5.4)$ to $b(\cdot, \cdot)=(\cdot, \cdot)_{L^{2}\left(\Omega_{R}\right)}+k \tilde{a}(\cdot, \cdot)$. For clarity of exposition we continue with the description of the FE discretization of (5.1) in the abstract framework of the previous section and explain in Section 5.1.3 how this applies to (4.1)-(4.2).

Let $\mathcal{V}_{N}=\operatorname{Span}\left\{\Phi_{i}\right\}_{i=1}^{N} \subset \mathcal{V}$ be a finite dimensional subspace of $\mathcal{V}$ of dimension $\operatorname{dim} \mathcal{V}_{N}=N$. Let $\mathcal{K}_{N}:=\mathcal{K} \cap \mathcal{V}_{N}$ and let $u_{N}$ be the solution of the following variational inequality

$$
\text { Find } u_{N} \in \mathcal{K}_{N}: \quad\left\langle B u_{N}, v-u_{N}\right\rangle_{\mathcal{V}} \geq\left\langle b_{l}, v-u_{N}\right\rangle_{\mathcal{V}} \quad \forall v \in \mathcal{K}_{N}
$$


Again, (5.5) is equivalent to the following fix-point interation

$$
\text { Given } u_{0, N} \in \mathcal{V}_{N} \text { define } u_{n+1, N}:=P_{\mathcal{K}_{N}}\left(u_{n, N}-\rho\left(B u_{n, N}-b_{l}\right)\right) \quad \forall n \geq 0
$$

where $P_{\mathcal{K}_{N}}$ denotes here the $\langle\cdot, \cdot\rangle_{\mathcal{V}}$ projection onto $\mathcal{K}_{N}$

Given $v \in \mathcal{V}$, find $P_{\mathcal{K}_{N}} v \in \mathcal{K}_{N}$ such that $\left\langle P_{\mathcal{K}_{N}} v, w-v\right\rangle_{\mathcal{V}} \geq\langle v, w-v\rangle_{\mathcal{V}} \quad \forall w \in \mathcal{K}_{N}$.

Let $\mathbf{H}$ denote the 'mass' matrix of $\langle\cdot, \cdot\rangle_{\mathcal{V}}$ in the basis $\mathcal{B}, \mathbf{B}$ the 'stiffness' matrix of the bilinear form $b(\cdot, \cdot)$ w.r. to $\mathcal{B}$ and $\underline{l}$ the 'load' vector, i.e.

$$
H_{i, j}:=\left\langle\Phi_{j}, \Phi_{i}\right\rangle_{\mathcal{V}}, \quad B_{i, j}=b\left(\Phi_{j}, \Phi_{i}\right), \quad l_{i}=l\left(\Phi_{i}\right) \quad 1 \leq i, j \leq N .
$$

'I'he fix-point iteration (5.6) is equivalent to:

Find $\underline{u}_{n+1} \in \underline{\mathcal{K}}_{N}:=\left\{\underline{v} \in \mathbb{R}^{N}: v:=\sum_{i=1}^{N} v_{i} \Phi_{i} \in \mathcal{K}_{N}\right\}$ such that

$$
\underline{u}_{n+1}^{\top} \mathbf{H}\left(\underline{v}-\underline{u}_{n+1}\right) \geq\left(\mathbf{H} \underline{u}_{n}-\rho\left(\mathbf{B} \underline{u}_{n}-\underline{l}\right)\right)^{\top}\left(\underline{v}-\underline{u}_{n+1}\right) \quad \forall \underline{v} \in \underline{\mathcal{K}}_{N},
$$

where $\underline{v}$ is the coefficient vector of $v$ with respect to the basis $\left\{\Phi_{i}\right\}_{i=1}^{N}$.

Let us denote by $(\cdot, \cdot)_{\mathbf{H}}$ the scalar product $(\underline{v}, \underline{w})_{\mathbf{H}}=\underline{v}^{\top} \mathbf{H} \underline{w}$ induced by the matrix $\mathbf{H}$. Then (5.7) can be written as:

Find $\underline{u}_{n+1} \in \underline{\mathcal{K}}_{N}$ such that

$$
\left(\underline{u}_{n+1}, \underline{v}-\underline{u}_{n+1}\right)_{\mathbf{H}} \geq\left(\mathbf{H} \underline{u}_{n}-\rho\left(\mathbf{B} \underline{u}_{n}-\underline{l}\right)\right)^{\top}\left(\underline{v}-\underline{u}_{n+1}\right) \quad \forall \underline{v} \in \underline{\mathcal{K}}_{N}
$$

which is the fixed point iteration applied to the bilinear form $b_{N}(\cdot, \cdot): \mathbb{R}^{N} \times \mathbb{R}^{N} \rightarrow \mathbb{R}$ and the linear form $l_{N}: \mathbb{R}^{N} \rightarrow \mathbb{R}$ given by

$$
b_{N}(\underline{v}, \underline{w})=\underline{v}^{\top} \mathbf{B} \underline{w}, \quad l_{N}(\underline{v})=\underline{v}^{\top} \underline{l} .
$$

The constants $C_{1, N}, C_{2, N}$ that enter into the choice of the relaxation parameter $0<\rho<$ $2 C_{2, N} /\left(C_{1, N}\right)^{2}$ and that determine the rate of convergence of the fix-point iteration (5.8) are $\left\|\mathbf{H}^{-1 / 2} \mathbf{B} \mathbf{H}^{-1 / 2}\right\|_{2}$ and $\lambda_{\min }\left(\left(\mathbf{H}^{-1 / 2}\left(\mathbf{B}+\mathbf{B}^{\top}\right) \mathbf{H}^{-1 / 2}\right)\right.$.

\subsubsection{Application to (4.1)-(4.2)}

The choice of the equivalent inner product $\langle\cdot, \cdot\rangle$ and of the matrix $\mathbf{H}$ in (5.8) will be used for preconditioning the fixed point iteration.

Denote by $\mathbf{A}^{\mathrm{E}}:=\mathbf{M}+k \tilde{\mathbf{A}}$. For a standard finite element basis and $\sigma \neq 0, \mathbf{A}^{\mathrm{E}}$ has a condition number of order $h^{-2}$ for small $h$ and fixed $k$. For the matrix $\mathbf{A}^{\mathrm{E}}$ in wavelet basis one can achieve uniformly bounded condition number.

Proposition 5.1 Assume that $0 \leq Y<2$ and fix $\kappa$ in (4.13) sufficiently large, but independent of $L$. Then the quantities

$$
\left\|\hat{\mathbf{A}}^{\mathrm{E}}\right\|_{2}:=\left\|\left(\mathbf{H}^{\mathrm{E}}\right)^{-1 / 2} \mathbf{A}^{\mathrm{E}}\left(\mathbf{H}^{\mathrm{E}}\right)^{-1 / 2}\right\|_{2}, \quad \lambda_{\min }\left(\left(\mathbf{H}^{\mathrm{E}}\right)^{-1 / 2}\left(\mathbf{A}^{\mathrm{E}}+\left(\mathbf{A}^{\mathrm{E}}\right)^{\top}\right)\left(\mathbf{H}^{\mathrm{E}}\right)^{-1 / 2}\right)
$$

where $\mathbf{H}^{\mathrm{E}}$ is a diagonal matrix with entries $H_{(j, l),(j, l)}^{\mathrm{E}}=1+k 2^{2 l}$, are bounded from above and below, respectively, in $L$ and $k$. In particular, the fixed point iteration (5.8) with $\mathbf{B}=\mathbf{A}^{\mathrm{E}}$ and $\mathbf{H}=\mathbf{H}^{\mathrm{E}}$ converges with rate $q<1$ independent of $k$ and $L$. 
Proof. Define $\hat{\mathbf{A}}^{\mathrm{E}}:=\left(\mathbf{H}^{\mathrm{E}}\right)^{-1 / 2} \mathbf{A}^{\mathrm{E}}\left(\mathbf{H}^{\mathrm{E}}\right)^{-1 / 2}$. By the norm equivalences (4.9) and the consistency condition (4.13) for sufficiently large $\kappa$ in (4.12) it holds

$$
\begin{aligned}
& C_{1}\|x\|_{\ell_{2}}^{2} \leq x^{\top} \mathbf{M} x, \quad x^{\top} \mathbf{M} y \leq C_{2}\|x\| \ell_{\ell_{2}}\|y\|_{\ell_{2}} \\
& C_{3}\|\mathbf{D} x\|_{\ell_{2}}^{2} \leq x^{\top} \tilde{\mathbf{A}} x, \quad x^{\top} \tilde{\mathbf{A}} y \leq C_{4}\|\mathbf{D} x\|_{\ell_{2}}\|\mathbf{D} y\|_{\ell_{2}}
\end{aligned}
$$

with $\mathbf{D}$ being the diagonal matrix with entries $D_{(j, l),(j, l)}=2^{l}$ and with constants $C_{j}$ independent of $L$. It follows that there exist some constants $C_{5}$ and $C_{6}>0$ independent of $L$ such that

$$
\begin{aligned}
& C_{5} x^{\top}(\mathrm{I}+k \mathbf{D}) x \leq x^{\top} \hat{\mathbf{A}}^{\mathrm{E}} x \\
& x^{\top} \hat{\mathbf{A}}^{\mathrm{E}} y \leq C_{6}\left[\|x\|_{\ell_{2}}\|y\|_{\ell_{2}}+k\|\mathbf{D} x\|_{\ell_{2}}\|\mathbf{D} y\|_{\ell_{2}}\right] \leq C_{6}\|(\mathrm{I}+k \mathbf{D}) x\|_{\ell_{2}}\|(\mathrm{I}+k \mathbf{D}) y\|_{\ell_{2}}
\end{aligned}
$$

which completes the proof.

The fix-point iteration (5.8) applied to (4.3)-(4.4) reads:

For $m=0,1, \ldots, T / M-1$ do:

For $n=0,1,2, \ldots$ until convergence:

Find $\underline{U}_{n+1, N}^{m+1} \in \underline{\mathcal{K}}_{0}$ such that

$$
\underline{U}_{n+1, N}^{m+1}{ }^{\top} \mathbf{H}^{\mathrm{E}}\left(\underline{v}-\underline{U}_{n+1, N}^{m+1}\right) \geq\left(\mathbf{H}^{\mathrm{E}} \underline{U}_{n, N}^{m+1}-\rho \mathbf{A}^{\mathrm{E}} \underline{U}_{n, N}^{m+1}+\rho\left(\mathbf{M} \underline{U}_{N}^{m}+k \underline{F}\right)\right)^{\top}\left(\underline{v}-\underline{U}_{n+1, N}^{m+1}\right) \quad \forall \underline{v} \in \underline{\mathcal{K}}_{0}
$$

Next $n$

Set $\underline{U}_{N}^{m+1}:=\underline{U}_{n, N}^{m+1}$

Next $m$

\begin{tabular}{|c|rrrrrrrrrrrrr|}
\hline$L \backslash \rho$ & 0.60 & 0.65 & 0.70 & 0.75 & 0.80 & 0.85 & 0.90 & 0.95 & 1.00 & 1.05 & 1.10 & 1.15 & 1.20 \\
\hline \hline 6 & 31 & 28 & 25 & 23 & 20 & 18 & 17 & 15 & 14 & 15 & 18 & 20 & 24 \\
\hline 7 & 31 & 28 & 25 & 23 & 20 & 18 & 17 & 15 & 14 & 15 & 18 & 20 & 24 \\
\hline 8 & 32 & 28 & 25 & 24 & 22 & 20 & 19 & 17 & 17 & 17 & 18 & 25 & 24 \\
\hline 9 & 29 & 34 & 28 & 28 & 26 & 22 & 20 & 22 & 17 & 20 & 24 & 33 & 28 \\
\hline
\end{tabular}

Table 1: Performance of the outer fix point iteration for $\sigma=0.2$. The CGMY parameters are $C=1.0, G=1.4, M=2.5$ and $Y=1.4$.

Remark 5.2 In the pure jump case, i.e., when $\sigma=0$, and for $Y \in(1,2)$ the bilinear form is continuous on $H_{0}^{Y / 2}\left(\Omega_{R}\right) \times H_{0}^{Y / 2}\left(\Omega_{R}\right)$ and satisfies the Garding inequality (4.16), see also Proposition 4.3 and Remark 4.4. By the norm equivalences (4.9) we define in this case $\mathbf{H}^{\mathrm{E}}$ as being the diagonal matrix with entries $H_{(j, l),(j, l)}^{\mathrm{E}}=1+k 2^{Y l}$ and the proof of Proposition 5.1 holds verbatim. When $Y \in[0,1]$ we applied the same numerical scheme and the perfomance of our solution algorithm turns out to be the same as reported for the case when $\sigma>0$ or $\sigma=0$ and $Y \in(1,2)$. 


\begin{tabular}{|c|rrrrrrrrrrrrr|}
\hline$L \backslash \rho$ & 0.60 & 0.65 & 0.70 & 0.75 & 0.80 & 0.85 & 0.90 & 0.95 & 1.00 & 1.05 & 1.10 & 1.15 & 1.20 \\
\hline \hline 6 & 24 & 21 & 19 & 17 & 16 & 16 & 13 & 13 & 13 & 12 & 15 & 19 & 23 \\
\hline 7 & 28 & 25 & 23 & 21 & 19 & 18 & 17 & 16 & 17 & 20 & 26 & 33 & 45 \\
\hline 8 & 30 & 27 & 24 & 23 & 22 & 20 & 19 & 18 & 22 & 27 & 32 & 40 & 59 \\
\hline 9 & 32 & 28 & 27 & 26 & 22 & 21 & 20 & 20 & 26 & 31 & 41 & 55 & 83 \\
\hline
\end{tabular}

Table 2: Performance of the outer fix point iteration for a pure jump Lévy process, i.e. $\sigma=0.0$. The CGMY parameters are $C=1.0, G=8.8, M=9.2$ and $Y=1.6$.

In Table 1 we study the rate of convergence of the outer iteration in dependence on $\rho$ and $L$ (the level of the FE discretization). More precisely, for a fixed time $t=0.5$ and a fixed time step $k=0.01$ we count the number $n$ of outer iterations needed for $\left\|\underline{U}_{n+1, N}-\underline{U}_{n, N}\right\|_{\mathbf{H}^{\mathrm{E}}}$ to fall below a given tolerance $t o l=10^{-8}$. The diffusion coefficient $\sigma$ is in this case $\sigma=0.2$ and the $\mathbf{H}^{E}$ projection corresponds, by the wavelet norm equivalences to the $H^{1}$ projection onto the convex conc of admissible solutions $\underline{\mathcal{K}}_{0}$

In Table 2 we repeat this experiment for a pure jump process (i.e. $\sigma=0$ ) with $Y=1.6$. The diagonal matrix $\mathbf{H}^{E}$ is now given by $H_{(j, l),(j, l)}^{\mathrm{E}}=1+k 2^{Y l}$, i.e. does not correspond to the Laplace matrix anymore. We observe that the number of outer iterations for e.g. $\rho=1.0$ is independent of $\sigma$ and of the choice of the discretization level parameter $L$.

\subsection{Realization of $P_{K}$}

It remains to solve the variational inequality (5.7). Note that $\mathbf{H}^{\mathrm{E}}$ is symmetric and, if the diagonal wavelet preconditioner is used, possibly diagonal. To realize the $\mathbf{H}^{\mathrm{E}}$ projection onto the convex cone $\underline{\mathcal{K}}_{0}$ we use a generalization of the Cryer algorithm [14].

\subsubsection{Generalized Cryer algorithm}

Let $\mathbf{H} \in \mathbb{R}^{N \times N}$ be any symmetric positive definite matrix and let $\mathbf{C} \in \mathbb{R}^{N \times N}$ be any invertible matrix. Specific choices of $\mathbf{H}$ and $\mathbf{C}$ will be given below. Consider the minimization problem

$$
\min _{\underline{u}} \underline{u}^{\top} \mathbf{H} \underline{u}-2 \underline{f}^{\top} \underline{u} \quad \text { subject to } \mathbf{C} \underline{u} \geq 0 \text { element-wise, }
$$

which corresponds to the LCP

$$
\begin{aligned}
& \text { Find } \underline{u} \in \mathbb{R}^{N} \text { such that } \mathbf{C} \underline{u} \geq 0 \\
& \mathbf{C}^{-\top}(\mathbf{H} \underline{u}-\underline{f}) \geq 0 \\
& \underline{u}^{\top}(\mathbf{H} \underline{u}-\underline{f})=0
\end{aligned}
$$

We use the following

Algorithm 5.3 Choose $\omega \in(0,2)$. Set $\underline{s}_{i}:=\mathbf{C}^{-1} \underline{e}_{i}, i=1, \ldots, N$.

o) Choose a starting vector $\underline{u}^{0}$ with $\mathbf{C} \underline{u}^{0} \geq 0$.

1) For $k=1, \ldots$ do (until convergence):

1.1) Set $\underline{u}_{0}^{k}:=\underline{u}^{k-1}$.

1.2) For $i=1, \ldots, N$ do:

1.2.1) $\operatorname{Set} \underline{r}_{i}^{k}=\left(\underline{s}_{i}^{\top} \mathbf{H} \underline{s}_{i}\right)^{-1} \underline{s}_{i}^{\top}\left[f-\mathbf{H} \underline{u}_{i-1}^{k}\right] \underline{s}_{i}$.

1.2.2) Choose the maximal $\tilde{\omega}_{i}^{k}$ with $\tilde{\omega}_{i}^{k} \leq \omega$, such that $\mathbf{C}\left(\underline{u}_{i-1}^{k}+\tilde{\omega}_{i}^{k} \underline{r}_{i}^{k}\right) \geq 0$.

1.2.3) Set $\underline{u}_{i}^{k}=\underline{u}_{i-1}^{k}+\tilde{\omega}_{i}^{k} \underline{r}_{i}^{k}$. 


\section{3) Next $i$. \\ 1.4) Set $\underline{u}^{k}:=\underline{u}_{N}^{k}$.}

2) Next $k$.

Lemma 5.4 Under the above assumptions, the algorithm converges to the solution of (5.10).

Proof. Let $G(\underline{u})=\underline{u}^{\top} \mathbf{H} \underline{u}-2 \underline{f}^{\top} \underline{u}$. Using symmetry of $\mathbf{H}$, one has

$$
G(\underline{u})-G(\underline{v})=(\underline{u}-\underline{v})^{\top} \mathbf{H}(\underline{u}-\underline{v})+2(\underline{u}-\underline{v})^{\top}(\mathbf{H} \underline{v}-\underline{f}) .
$$

With

we get

$$
\left(\underline{r}_{i}^{k}\right)^{\top}\left(\mathbf{H} \underline{u}_{i-1}^{k}-\underline{f}\right)=-\frac{\underline{s}_{i}^{\top} \mathbf{H} \underline{s}_{i}}{\underline{s}_{i}^{\top} \underline{s}_{i}}\left(\underline{r}_{i}^{k}\right)^{\top} \underline{r}_{i}^{k}
$$

$$
G\left(\underline{u}_{i}^{k}\right)-G\left(\underline{u}_{i-1}^{k}\right)=\left(\tilde{\omega}_{i}^{k}\right)^{2}\left(\underline{r}_{i}^{k}\right)^{\top} \mathbf{H} \underline{r}_{i}^{k}-2 \tilde{\omega}_{i}^{k} \frac{\underline{s}_{i}^{\top} \mathbf{H} \underline{s}_{i}}{\underline{s}_{i}^{\top} \underline{s}_{i}}\left(\underline{r}_{i}^{k}\right)^{\top} \underline{r}_{i}^{k} .
$$

Since $\underline{r}_{i}^{k}$ is a multiple of $\underline{s}_{i}$, equation (5.11) reads

$$
G\left(\underline{u}_{i}^{k}\right)-G\left(\underline{u}_{i-1}^{k}\right)=-\tilde{\omega}_{i}^{k}\left(2-\tilde{\omega}_{i}^{k}\right)\left(\underline{r}_{i}^{k}\right)^{\top} \mathbf{H} \underline{r}_{i}^{k} \leq 0,
$$

the last inequality due to $\tilde{\omega}_{i}^{k} \in[0, \omega] \subset[0,2)$.

Hence, the sequence $\left\{G\left(\underline{u}_{i}^{k}\right)\right\}$ is monotonically decreasing. By positivity of $\mathbf{H}, G$ is strictly convex and bounded below. It follows, that $G\left(\underline{u}_{i}^{k}\right) \rightarrow G$ from above.

Using

$$
\left(\underline{r}_{i}^{k}\right)^{\top} \mathbf{H} \underline{r}_{i}^{k}=\frac{1}{\left(\tilde{\omega}_{i}^{k}\right)^{2}}\left(\underline{u}_{i}^{k}-\underline{u}_{i-1}^{k}\right)^{\top} \mathbf{H}\left(\underline{u}_{i}^{k}-\underline{u}_{i-1}^{k}\right)
$$

in case $\tilde{\omega}_{i}^{k} \neq 0$, we infer

$$
G\left(\underline{u}_{i}^{k}\right)-G\left(\underline{u}_{i-1}^{k}\right) \leq\left(1-\frac{2}{\omega}\right)\left(\underline{u}_{i}^{k}-\underline{u}_{i-1}^{k}\right)^{\top} \mathbf{H}\left(\underline{u}_{i}^{k}-\underline{u}_{i-1}^{k}\right) \leq-c\left\|\underline{u}_{i}^{k}-\underline{u}_{i-1}^{k}\right\|^{2}
$$

with some $c>0$, i. e.

$$
\left\|\underline{u}_{i}^{k}-\underline{u}_{i-1}^{k}\right\|^{2} \leq C\left[G\left(\underline{u}_{i-1}^{k}\right)-G\left(\underline{u}_{i}^{k}\right)\right] .
$$

Hence, $\underline{u}_{i}^{k}$ converges as well, say, to $\underline{u} \in \mathbb{R}^{N}$. By continuity of the algorithm, $\underline{u}$ is a fixed point. It remains to show, that $\underline{u}$ solves the minimization problem (5.10).

Let

$$
K=\left\{\underline{u} \in \mathbb{R}^{N}: \mathbf{C} \underline{u} \geq 0\right\} .
$$

The constraint set $K$ is the mapped positive orthant $K_{0}=\left\{\underline{u} \in \mathbb{R}^{N}: \underline{u} \geq 0\right\}$, i.e. $K=\mathbf{C}^{-1} K_{0}$. Let $\underline{x} \in \mathbb{R}^{N}$ and let $\underline{h}_{i}, i=1, \ldots, n$, be a set of directions. If $G^{\prime}(\underline{x}) \underline{h}_{i}$, the directional derivative of $G$ at $\underline{x}$ in the direction $\underline{h}_{i}$, is non-negative for all $i=1, \ldots, n$, then for every set of nonnegative numbers $\alpha_{i}, i=1, \ldots, n$, it holds $G\left(\underline{x}+\sum \alpha_{i} \underline{h}_{i}\right) \geq G(\underline{x})$, or, equivalently, $\underline{x}$ is the minimizer of $G$ over the cone spanned by the directions $\underline{h}_{i}$. To see this, set $\underline{h}:=\sum \alpha_{i} \underline{h}_{i}$ and note, that by linearity of $G^{\prime}$ we have $G^{\prime}(\underline{x}) \underline{h} \geq 0$. Taking into account that $G$, restricted to the line $\{\underline{x}+\alpha \underline{h}, \alpha \in \mathbb{R}\}$, is a convex funtion in $\alpha$, the claim follows.

Now the assertion, that $\underline{u}$ is the solution to $(5.10)$, easily follows. We call a direction $\underline{d} \in \mathbb{R}^{N}$ pointing outwards of $K$ at $x$, if for every $\varepsilon>0$ the point $\underline{x}+\varepsilon d \notin K$. (Note, that for $\underline{x} \in K$ by convexity of $K$ the fact $\underline{x}+\varepsilon_{0} d \notin K$ implies $\underline{x}+\varepsilon d \notin K$ for every $\varepsilon \geq \varepsilon_{0}$.)

Set $S=\left\{ \pm \underline{s}_{i}, i=1, \ldots, N\right\}$ and consider the set

$$
S(\underline{u}):=\{\underline{s} \in S: \underline{s} \text { is not pointing outwards of } K \text { at } \underline{u}\} .
$$


For $s \in S(u)$ by stationarity of the algorithm, it holds $G^{\prime}(\underline{u}) \underline{s} \geq 0$. On the other hand, the convex cone $C S(\underline{u})$ generated by $S(\underline{u})$ satisfies

$$
K \subset C S(\underline{u})
$$

By the above reasoning, $\underline{u}$ is the minimizer of $G$ over $C S(\underline{u})$, and therefore the minimizer of $G$ over $K$.

There seem to be no convergence rates available for Cryer's algorithm. Multilevel techniques [27] promise an $O(N \log (N))$ efficiency, but in the parabolic examples considered below, the direct application of Cryer's algorithm turned out to be superior, since the initial values taken from the previous steps are very close to the respective solutions. In our experiments, the number of inner itcrations was usually of the same size as the number of iterations in the outer (Stampacchia) iteration.

\section{$6 \quad$ Numerical Results}

We present in this section extensive numerical experiments to illustrate the flexibility of our code with respect to the choice of the price process parameters and of the discretization parameters. In all experiments below the level $L$ for the wavelet resolution is $L=11$.

In the first example shown on Figure 3 we take $K=500, T=0.5, r=0.4, \sigma=0.2$ and the following CGMY parameters: $C=1, G=1.4, M=2.5$ and $Y=1.4$. We compare the value of the American put option with that of the European one for the same set of parameters. The early exercise boundary of the American contract is plotted on the right.

In Figure 4 we display the prices of American put options with respect to different time horizons $T=0.1,0.25,0.5$. The price process is a pure jump CGMY Lévy process with parameters $C=1.0, G=8.8, M=9.2$ and $Y=1.6$. In Figure 5 we take $\mathrm{VG}$ as price process, i.e., $\sigma=0.0$ and $Y=0.0$.

In [10] it is shown that in the case of perpetual American put, i.e., for $T=\infty$ (equivalently, for the stationary variant of (3.4)-(3.7)), the principle of smooth fit may fail in the case of infinite intensily pure junp Lévy processes. From the resulis of [10] it follows in particular liad for the family of CGMY Lévy processes, the principle of smooth fit may fail if $Y \in(0,1)$, but also that the smooth fit condition always holds for $Y \in[1,2)$. We emphasize that we do not rely on the principle of smooth fit within our numerical scheme. Our numerical experiments also reveal that in the case $Y \in[1,2)$ the smooth fit condition appears to hold (see Figures 3, 4) whereas it fails for the case of the VG process in Figure 5.

Studying sensitivity of prices with respect to parameters is of great importance and we illustrate this feature in Figure 6, where we fix all parameters except $Y$ and study how the exercise boundary depends on $Y$.

Our last example concerns an American butterfly option. Its pay-off is given by

$$
\left(S-K_{1}\right)_{+}-2\left(S-\left(K_{1}+K_{2}\right) / 2\right)_{+}+\left(S-K_{2}\right)_{+}
$$

and is constructed by holding a long position in two calls with strikes at $K_{1}$ and $K_{2}$ and a short position in two calls struck at $\left(K_{1}+K_{2}\right) / 2$. Note that the pay-off function is not convex anymore, a feature that is often exploited to speed up convergence of certain algorithms [7]. In [7] an algorithm for evaluating the American put option within the classical Black-Scholes framework in linear computational complexity with respect to the number of grid points is proposed. The method relies however on the monotonicity of the exercise boundary and on the band structure of the matrix of the LCP. We emphasize that our implementation does not 
rely on any topological assumption on the free boundary as e.g., graph-like and monotone. We plot in Figure 7 the option value of an American butterfly option with $K_{1}=3$ and $K_{2}=10$ and the early exercise boundary for this case.
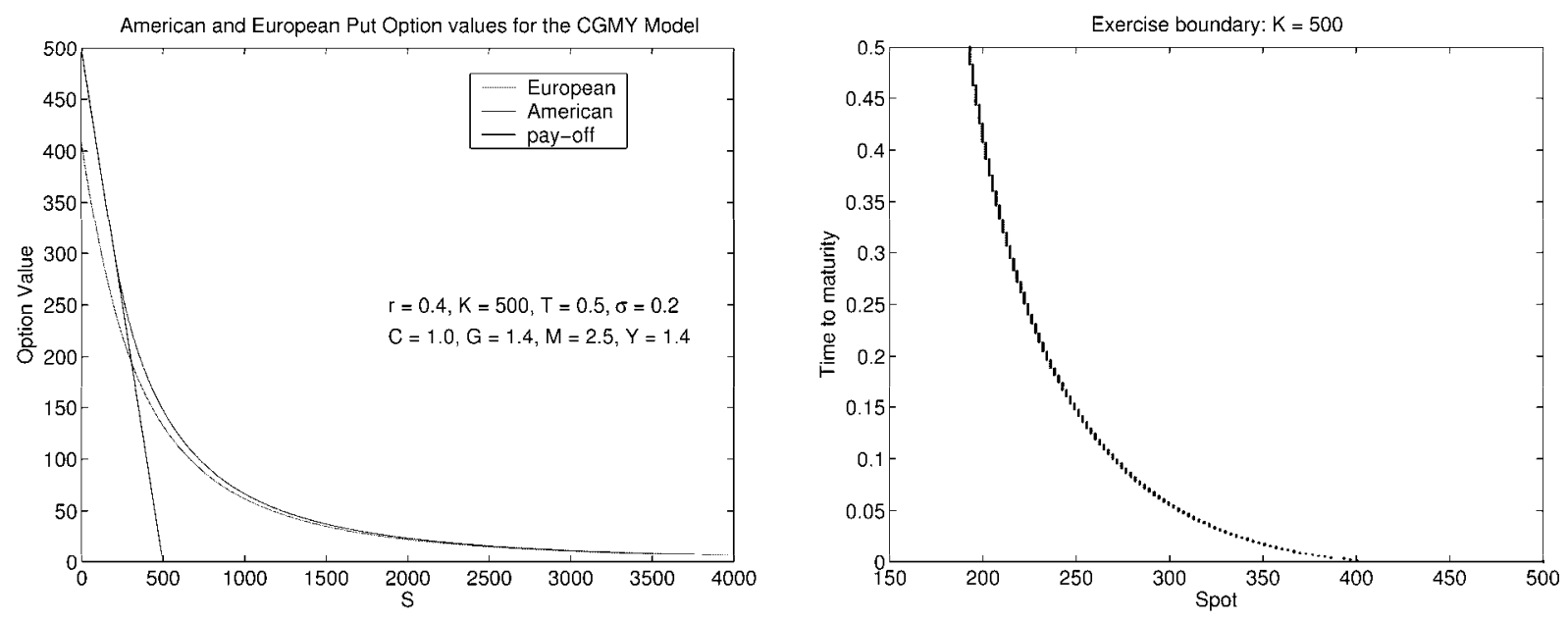

Figure 3: American vs. European and the exercise boundary
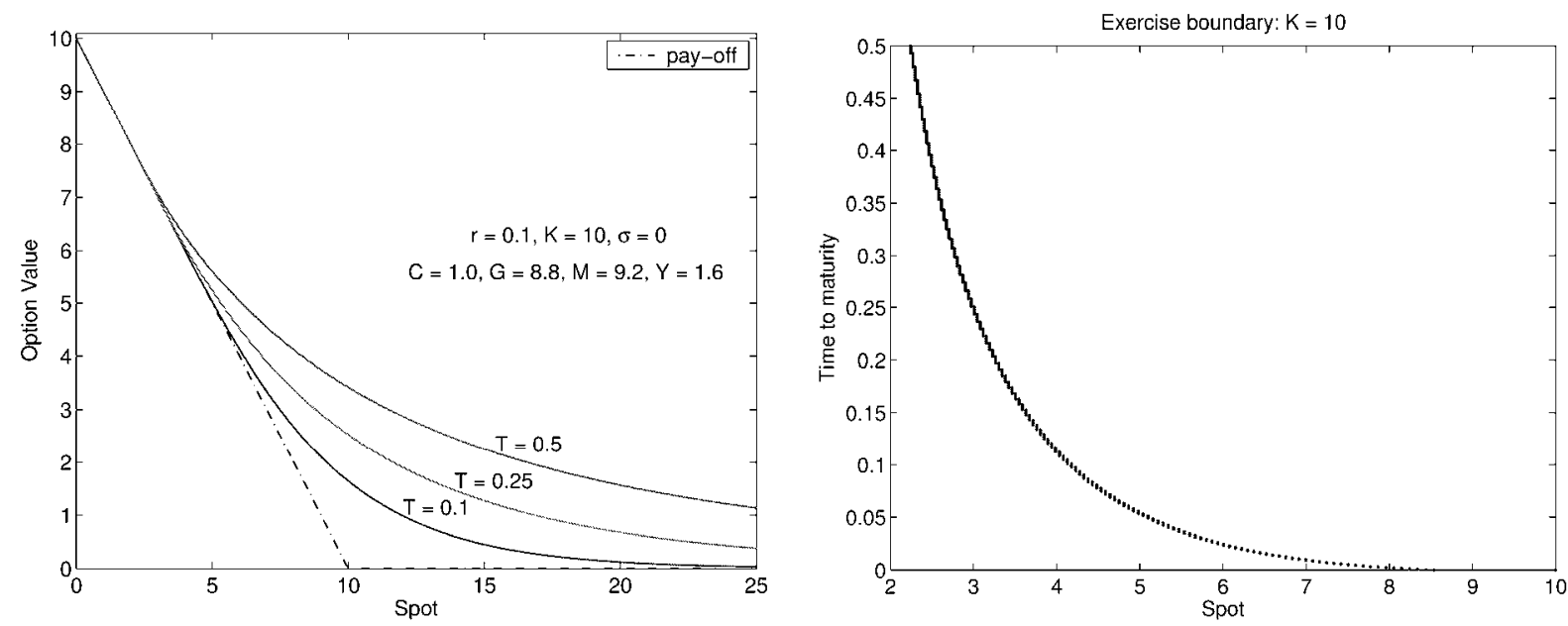

Figure 4: American Put option values for different maturities and for a pure jump Lévy process of CGMY type with $C=1.0, G=8.8, M=9.2$ and $Y=1.6$.

\section{A Proof of Theorem 3.4}

Here, we give the proof of Theorem 3.4. We split the bilinear form $a^{\eta}$ into the following expressions

$$
a^{\eta}=a_{1}^{\eta}+a_{2}^{\eta}+a_{3}^{\eta}
$$



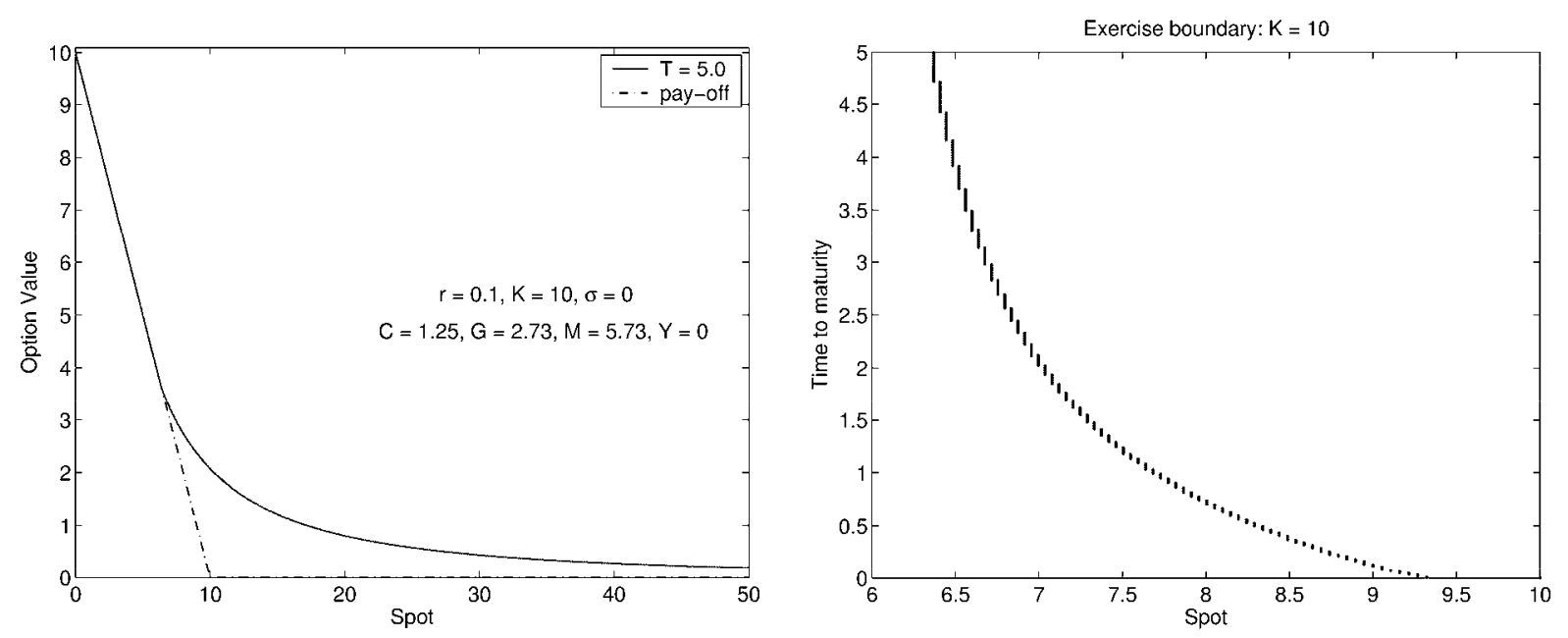

Figure 5: Failure of the smooth pasting condition for VG as price process.
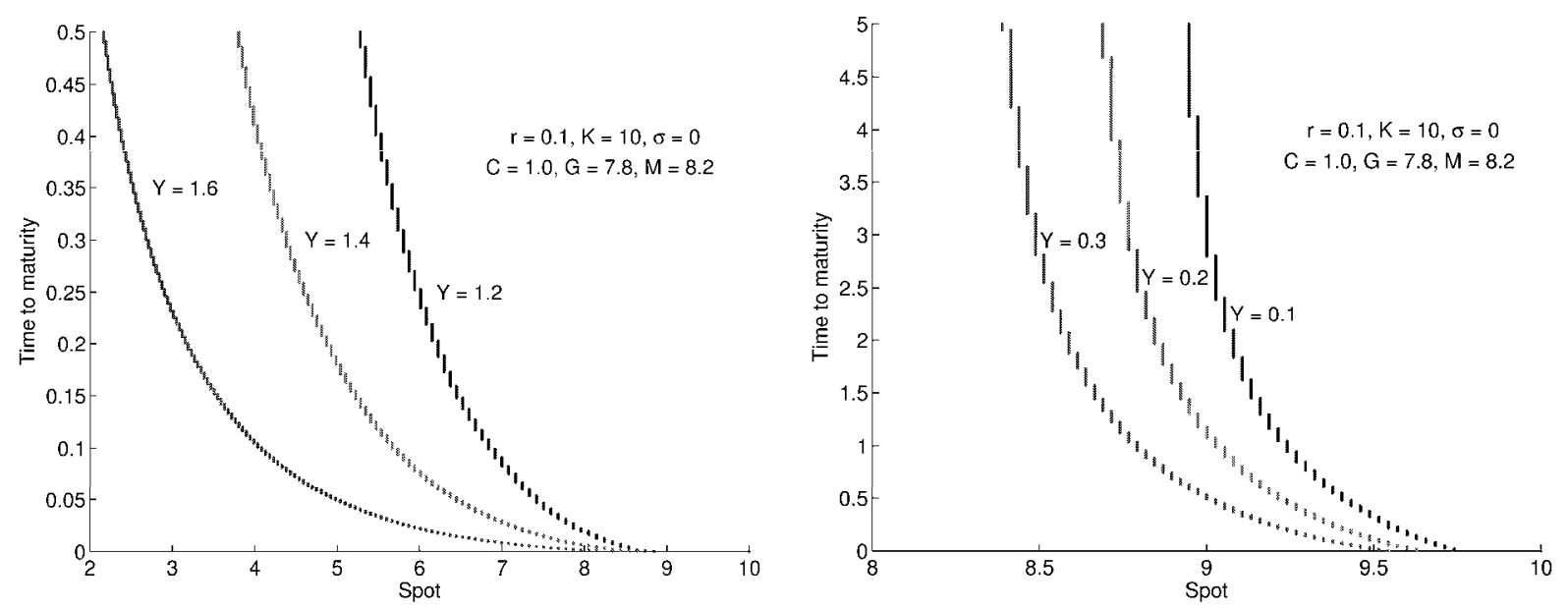

Figure 6: Sensitivity of the exercise boundary with respect to parameter $Y$ in the CGMY model.

where

$$
\begin{aligned}
a_{1}^{\eta}(\varphi, \phi)= & \frac{\sigma^{2}}{2} \int_{\mathbb{R}} \frac{\mathrm{d} \varphi}{\mathrm{d} x}(x) \frac{\mathrm{d} \phi}{\mathrm{d} x}(x) e^{2 \eta(x)} \mathrm{d} x-r \int_{\mathbb{R}}\left(\frac{\mathrm{d} \varphi}{\mathrm{d} x}(x)-\varphi(x)\right) \phi(x) e^{2 \eta(x)} \mathrm{d} x \\
& +\int_{\mathbb{R}} \frac{\mathrm{d} \varphi}{\mathrm{d} x}(x) \phi(x)\left(\frac{\sigma^{2}}{2}\left(2 \frac{\mathrm{d} \eta}{\mathrm{d} x}(x)+1\right)+\int_{\mathbb{R}}\left(e^{y}-1-y \chi_{\{|y| \leq 1\}}(y) k(y) \mathrm{d} y\right)\right) e^{2 \eta(x)} \mathrm{d} x \\
a_{2}^{\eta}(\varphi, \phi)= & -\int_{\mathbb{R}} \int_{\mathbb{R}} \int_{0}^{1} \mathrm{~d} \theta \frac{\mathrm{d} \varphi}{\mathrm{d} x}(x+\theta y) \phi(x) e^{2 \eta(x)} y \chi_{\{|y|>1\}}(y) k(y) \mathrm{d} y \mathrm{~d} x \\
a_{3}^{\eta}(\varphi, \phi)= & -\int_{\mathbb{R}} \int_{\mathbb{R}} \int_{0}^{1} \mathrm{~d} \theta \int_{0}^{\theta} \mathrm{d} \theta^{\prime} \frac{\mathrm{d}^{2} \varphi}{\mathrm{d} x^{2}}\left(x+\theta^{\prime} y\right) y^{2} \phi(x) e^{2 \eta(x)} \chi_{\{|y| \leq 1\}}(y) k(y) \mathrm{d} y \mathrm{~d} x .
\end{aligned}
$$



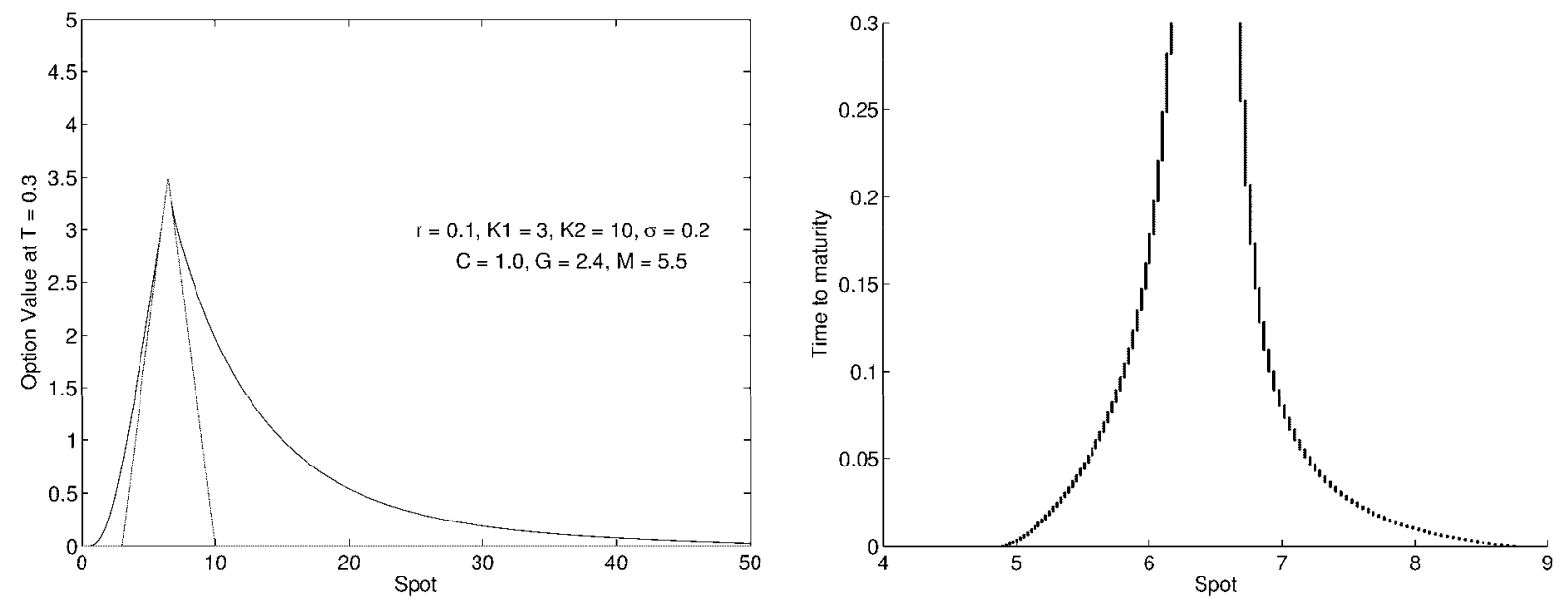

Figure 7: American butterfly

Clearly, for all $\varphi, \phi \in H_{\eta}^{1}(\mathbb{R})$ it holds

$$
\begin{aligned}
\left|a_{1}^{\eta}(\varphi, \phi)\right| \leq & C\left(\left\|\eta^{\prime}\right\|_{L^{\infty}(\mathbb{R})}\right)\left\|\frac{\mathrm{d} \varphi}{\mathrm{d} x}\right\|_{L_{\eta}^{2}(\mathbb{R})}\left\|\frac{\mathrm{d} \phi}{\mathrm{d} x}\right\|_{L_{\eta}^{2}(\mathbb{R})} \\
a_{1}^{\eta}(\varphi, \varphi) \geq & \frac{\sigma^{2}}{2}\left\|\frac{\mathrm{d} \varphi}{\mathrm{d} x}\right\|_{L_{\eta}^{2}(\mathbb{R})}^{2}+r\|\varphi\|_{L_{\eta}^{2}(\mathbb{R})}^{2} \\
& -\left(\frac{\sigma^{2}}{2}\left(2\left\|\eta^{\prime}\right\|_{L^{\infty}(\mathbb{R})}+1\right)+\mid \int_{\mathbb{R}} e^{y}-1-y \chi\{|y| \leq 1\}\right. \\
& (y) k(y) \mathrm{d} y \mid)\left\|\frac{\mathrm{d} \varphi}{\mathrm{d} x}\right\|_{L_{\eta}^{2}(\mathbb{R})}\|\varphi\|_{L_{\eta}^{2}(\mathbb{R})} .
\end{aligned}
$$

If we insert $e^{\eta(x+\theta y)} e^{-\eta(x+\theta y)}$ in the definition of $a_{2}^{\eta}$ and use the hypothesis on the weighting exponent $\eta$ we obtain that

$$
\left|a_{2}^{\eta}(\varphi, \phi)\right| \leq C\left\|\frac{\mathrm{d} \varphi}{\mathrm{d} x}\right\|_{L_{\eta}^{2}(\mathbb{R})}\|\phi\|_{L_{\eta}^{2}(\mathbb{R})}
$$

The remaining part $a_{3}^{\eta}$ needs a more careful inspection. It can be shown that for any $\varepsilon>0$ there exists a constant $C(\varepsilon)>0$ such that

$$
\left|a_{3}^{\eta}(\varphi, \phi)\right| \leq \varepsilon\left\|\frac{\mathrm{d} \varphi}{\mathrm{d} x}\right\|_{L_{\eta}^{2}(\mathbb{R})}\left\|\frac{\mathrm{d} \phi}{\mathrm{d} x}\right\|_{L_{\eta}^{2}(\mathbb{R})}+C(\varepsilon)\left\|\frac{\mathrm{d} \varphi}{\mathrm{d} x}\right\|_{L_{\eta}^{2}(\mathbb{R})}\|\phi\|_{L_{\eta}^{2}(\mathbb{R})} .
$$

To prove (A.1) we write $a_{3}^{\eta}(\varphi, \phi)$ in the following form

$$
\begin{aligned}
a_{3}^{\eta}(\varphi, \phi) & = \\
& \int_{\mathbb{R}} \int_{\mathbb{R}} \int_{0}^{1} \mathrm{~d} \theta \int_{0}^{\theta} \mathrm{d} \theta^{\prime} \frac{\mathrm{d} \varphi}{\mathrm{d} x}\left(x+\theta^{\prime} y\right)\left(\frac{\mathrm{d} \phi}{\mathrm{d} x}(x)+2 \eta^{\prime}(x) \phi(x)\right) e^{2 \eta(x)} y^{2} \chi_{\{|y| \leq \delta\}}(y) k(y) \mathrm{d} y \mathrm{~d} x \\
& -\int_{\mathbb{R}} \int_{\mathbb{R}} \int_{0}^{1} \mathrm{~d} \theta \frac{\mathrm{d} \varphi}{\mathrm{d} x}(x+\theta y) e^{\eta(x+\theta y)} \phi(x) e^{\eta(x)} e^{-\eta(x+\theta y)+\eta(x)} y \chi_{\{\delta \leq|y| \leq 1\}}(y) k(y) \mathrm{d} y \mathrm{~d} x \\
& +\int_{\mathbb{R}} \int_{\mathbb{R}} \frac{\mathrm{d} \varphi}{\mathrm{d} x}(x) \phi(x) e^{2 \eta(x)} y \chi_{\{\delta \leq|y| \leq 1\}}(y) k(y) \mathrm{d} y \mathrm{~d} x .
\end{aligned}
$$

Since $\int_{\{|y| \leq 1\}} y^{2} k(y) \mathrm{d} y<+\infty$, given $\varepsilon>0$, one can choose $\delta=\delta(\varepsilon)$ sufficiently small such that (A.1) holds. 


\section{References}

[1] R.A. Adams, Sobolev Spaces, Academic Press, New York 1978.

[2] L. Andersen and J. Andreasen, Jump-diffusion processes: Volatility smile fitting and numerical methods for option pricing, Review of Derivatives Research 4(2000) 231-262.

[3] O.E. Barndorff-Nielsen, Exponentially decreasing distributions for the logarithm of particle size, Proceedings of the Royal Society London A 353 (1977) 401-419.

[4] A. Bensoussan, J.-L. Lions, Impulse control and quasi-variational inequalities, GauthierVillars, Paris 1984.

[5] J. Bertoin, Lévy processes, Cambridge Univ. Press, 1996.

[6] F. Black, M. Scholes, The Pricing of Options and Corporate Liabilities, Journal of Political Economy 81 (1973) 637-654.

[7] A. Borici, H.-J. Lüthi, Pricing American Put Options by Fast Solutions of the Linear Complementarity Problem, in Computational Methods in Decision-Making, Economy and Finance, Ed. E. J. Kontoghiorghes, B. Rustem, S. Siokos, Kluwer Academic Publishers 2002.

[8] S. Boyarchenko, S. Levendorski, Barrier Options and Touch-and-Out Options under Regular Lévy Processes of Exponential Type, to appear in The Annals of Applied Probability, 2002.

[9] S. Boyarchenko, S. Levendorski, Option pricing for truncated Lévy processes, Int. Journ. Theor. and Appl. Finance (2000).

[10] S. Boyarchenko, S. Levendorski, Perpetual American Options under Lévy Processes, SIAM J. Control Optim. (2002) Vol. 40, No. 6, 1663-1696.

[11] P. Carr, H. Geman, D.B. Madan, The Fine Structure of Asset Returns: An Empirical Investigation, Journal of Business 2002.

[12] P. Carr, D. Madan, Option valuation using the FFT, J. Comp. Finance 2 (1999) 61-73.

[13」 T. Chan, Pricing Contingent Claims on Stocks Driven by Lévy Processes, The Annals of Applied Probability, (1999) Vol. 9, No. 2, 504-528.

[14] C.W. Cryer, The solution of a quadratic programming problem using systematic overrelaxation, SIAM J. Control (9) (1971) 385-392.

[15] F. Delbaen, W. Schachermayer, The Variance-Optimal Martingale Measure for Continuous Processes, Bernoulli 2 (1996), no. 1, 81-105.

[16] F. Delbaen, P. Grandits, T. Rheinländer, D. Samperi, M. Schweizer, C. Stricker, Exponential Hedging and Entropic Penalties, Mathematical Finance, Vol.12, No.2, 99-123.

[17] E. Eberlein, Application of generalized hyperbolic Lévy motions to finance in Lévy Processes: Theory and Applications, O.E. Barndorff-Nielsen, T. Mikosch, and S. Resnick (Eds.), Birkhäuser (2001), 319-337.

[18] E. Eberlein, U. Keller, Hyperbolic distributions in finance, Bernoulli 1 (1995) 281-299. 
[19] H. Föllmer, M. Schweizer, Hedging of Contingent Claims under incomplete information, Applied Stochastic Analysis, M.H.A. Davis and R.J. Elliot, Eds., (1991) Gordon and Breach New York, 389-414.

[20] R. Glowinski, J.L. Lions and R. Trémolières, Numerical Analysis of Variational Inequalities, North-Holland Amsterdam 1981.

[21] G.H. Golub, C.F. Van Loan, Matrix Computations (1996) Johns Hopkins University Press.

[22] J. Jacod, A.N. Shiryaev, Limit Thenrems for Stochastic, Processes, Springer, Berlin 2002.

[23] P. Jaillet, D. Lamberton, B. Lapeyre, Variational Inequalities and the Pricing of American Options Acta Applicandae Mathematicae 21 (1990) 263-289.

[24] C. Johnson, A convergene result for an approximation of a parabolic variational inequality, SIAM J. Numer. Anal. 13 (1976) 599-606.

[25] I. Karatzas, S.E. Shreve, Methods of Mathematical Finance, Springer-Verlag, Berlin Heidelberg New York 1998.

[26] I. Koponen, Analytic approach to the problem of convergence of truncated Lévy flights towards the Gaussian stochastic process, Phys. Rev. E 52 (1995) 1197-1199.

[27] R. Kornhuber, Monotone multigrid methods for variational inequalities I., Numerische Mathematik 69 (1994) $167-184$.

[28] D. Lamberton, B. Lapeyre, Introduction to Stochastic Calculus Applied to Finance, Chapman \& Hall 1997.

[29] J.L. Lions, G. Stampacchia, Variational inequalities, Comm. Pure Appl. Math. 20 (1967) 493-519.

[30] D.B. Madan, P. Carr, E. Chang, The Variance Gamma Process and Option Pricing, European Finance Review 2 (1998) 79-105.

[31] D.B. Madan, E. Seneta, The variance gamma (V.G.) model for share market returns, Journal of Business 63(4) (1990) 511-524.

[32] A.-M. Matache, T. von Petersdorff, C. Schwab, Fast Deterministic Pricing of Options on Lćvy Driven Asscts, submittcd; available also as Rescarch Report 2002-11 Scminar for Applied Mathematics, ETH Zürich

http://www. sam.math.ethz.ch/reports/details/include.shtml?2002/2002-11.html

[33] M. Musiela, M. Rutkowski, Martingale methods in financial modelling, Springer Verlag, Berlin Heidelberg New York 1997.

[34] T. von Petersdorff, C. Schwab, Wavelet-discretizations of parabolic integro-differential equations, To appear in SIAM J. Numer. Anal. Available also as Research Report No. 2001-07, Seminar for Applied Mathematics, ETH Zürich. http://www.sam.math.ethz.ch/reports/details/include.shtml?2001/2001-07.html

[35] K.-I. Sato, Lévy Processes and Infinitely Divisible Distributions, Cambridge University Press, 1999.

[36] A.N. Shiryaev, Essentials of Stochastic Finance. Facts, models, theory, World Scientific, Singapore New Jersey London Hong Kong 1999. 